\title{
Spectral properties of electrostatic drift wave turbulence in the laboratory and the ionosphere
}

\author{
H. L. Pécseli \\ University of Oslo, Department of Physics, P.O. Box 1048 Blindern, 0316 Oslo, Norway \\ Correspondence to: H. L. Pécseli (hans.pecseli@fys.uio.no)
}

Received: 10 March 2015 - Revised: 22 May 2015 - Accepted: 26 June 2015 - Published: 23 July 2015

\begin{abstract}
Low-frequency electrostatic drift wave turbulence has been studied in both laboratory plasmas and in space. The present review describes a number of such laboratory experiments together with results obtained by instrumented spacecraft in the Earth's near and distant ionospheres. The summary emphasizes readily measurable quantities, such as the turbulent power spectra for the fluctuations in plasma density, potential and electric fields. The agreement between power spectra measured in the laboratory and in space seems to be acceptable, but there are sufficiently frequent counterexamples to justify a future dedicated analysis, for instance by numerical tools, to explain deviations. When interpreting spectra at low ionospheric altitudes, it is necessary to give attention to the DC ionospheric electric fields and the differences in the physics of electron-ion collisions and collisions of charged particles with neutrals for cases with significant Hall drifts. These effects modify the drift wave spectra. A dedicated laboratory experiment accounted for some of these differences.
\end{abstract}

Keywords. Electromagnetics (plasmas)

\section{Introduction}

Turbulence in neutral flows has been studied extensively, in part because of its significance for industry, the environment, etc., but also because of the theoretical intricacies which the phenomenon represents. An understanding of turbulent flows is important for weather forecasting, environmental pollution, windmill design, the transport of material by water flows in industrial plants, cooling and many other applications (Tennekes and Lumley, 1972; Hinze, 1975). Enhanced density fluctuations in the Earth's ionosphere can contribute to the scattering of radiation or electromagnetic waves (Bekefi, 1966), such as radio communication or waves used for Global Positioning Systems (GPS). In plasmas, in particular, turbulent transport will be mixing regions with different compositions and parameters, eroding density gradients especially (Taylor and McNamara, 1971; Misguich et al., 1987). The problem is of interest in nature as well as in laboratory experiments, including fusion plasma confinement. Low-frequency electrostatic drift waves have been found to be particularly important. They are often called "universal instabilities" since they are associated with plasma density gradients (Chen, 1984), or more generally plasma pressure gradients, which are unavoidable in the laboratory as well as in naturally occurring plasmas and which are, hence, universal. An inhomogeneous plasma is not in perfect thermodynamic equilibrium and the pressure tends to expand the plasma. The expansion can provide free energy for driving an instability (Chen, 1965d, e, 1984). The direction of linear drift wave propagation is almost perpendicular to an ambient magnetic field $\boldsymbol{B}$, satisfying an inequality $u_{\text {Thi }} \ll \omega / k_{\|} \ll u_{\text {The }}$ in terms of the ion and electron thermal velocities $u_{\text {Thi }}$ and $u_{\text {The }}$, respectively, with $\omega / k_{\|}$being the $\boldsymbol{B}$ parallel component of the phase velocity. The wave frequencies $\omega$ are much below $\Omega_{\text {ci }}$, the ion cyclotron frequency. At these low frequencies, the ion motion $\perp \boldsymbol{B}$ is well described by the $\widetilde{\boldsymbol{E}} \times \boldsymbol{B} / B^{2}$ velocity corrected by the lowest-order ion polarization drift, with $\widetilde{E}$ being the fluctuating electrostatic field. Compressibility arises only by the small contribution from the polarization drift, but the density fluctuations can be significant since the waves propagate with the electron diamagnetic drift in a density gradient. Even incompressible motion induces local density variations when plasma moves in a density gradient $\nabla n_{0} \perp \boldsymbol{B}$. Electrostatic drift waves are typically found for $\beta \leq m / M$, the electron / ion mass ratio, with $\beta$ being the ratio of plasma and magnetic field pres- 
sures (Chen, 1984). For larger plasma $\beta$ values we find a coupling to Alfvén waves. Turbulence in, for instance, the high- $\beta$ plasma of the solar wind requires a different approach (Tu and Marsch, 1995; Bruno and Carbone, 2005).

Electrostatic drift waves become unstable when a certain mechanism inhibits the free flow of electrons along magnetic field lines from wave crest to wave trough. Electron-ion collisions have this effect, but in kinetic models electrons in resonance with the drift waves will also induce linear instability, although with modest growth rates. Plasma currents can enhance the instability (Kadomtsev, 1965). The theoretic results for the linear instability (the resistive instabilities, in particular) of drift waves have been confirmed to good accuracy by a number of laboratory experiments (Hendel et al., 1968; Rowberg and Wong, 1970; Rogers and Chen, 1970; Schlitt and Hendel, 1972), mostly in Q machines (Motley, 1975; Pécseli, 2012). The fully developed saturated turbulent state of these instabilities is usually found to be nearly two-dimensional in the plane perpendicular to $\boldsymbol{B}$. One possible way of characterizing such turbulent states is represented by the mean square fluctuation levels and the entire normalized frequency-wave-vector-varying power spectra of fluctuating quantities, i.e. plasma density, potential or electric fields. The corresponding correlation functions represent one alternative. These results would contain all the available statistical information for a Gaussian random process with zero mean.

Spectra are often easy to measure, and many studies both in the laboratory and in space have reported such results. It is a possibility that turbulent power spectra can be used to identify some of the underlying mechanisms and instabilities that give rise to the enhanced fluctuation levels. For the time being, this remains speculative, but there seems to be some basis for the argument. Drift wave turbulence, when it is fully developed, seems to posses certain universal features, best found by considering a fit in terms of a power law in a wave number representation. The present review will discuss this possibility, illustrated by results from laboratory and from space observations, with reference also to some of the analytical results. Comparatively smaller attention is given to numerical results in order to limit the exposition.

Higher-order spectral methods, such as bispectra (Kim and Powers, 1979; Larsen et al., 2002; Yamada et al., 2008) and their generalizations, represent valuable tools for obtaining information that cannot be revealed by power spectra, accounting for non-Gaussian, intermittent features (Rollefson, 1978). Unfortunately the increase in information comes at a price, and higher-order spectra require increasingly longer records. Space measurements are often limited in time, and the accuracy of higher-order spectral studies is often limited. For laboratory studies the conditions can be much better.

Following a brief summary of some basic results concerning neutral flow turbulence, the present review will emphasize drift wave turbulence supplemented by a shorter discussion related to current-driven ion acoustic instabilities. This approach may appear unduly restrictive, but for magnetized low- $\beta$ plasmas, there are in reality rather few candidates for low-frequency turbulence with $\omega \ll \Omega_{\mathrm{ci}}$. Current-driven instabilities can be excited at low frequencies, and velocity shear instabilities can also give rise to enhanced levels of low-frequency fluctuations. As far as the velocity shear instability perpendicular to magnetic fields is concerned, it will here be argued that on small scales, the turbulent spectrum is insensitive to the energy source: on this level there is no need to distinguish drift wave instabilities from those caused by velocity shears transverse to magnetic fields. The energy cascade on the small turbulence scales can be expected to be independent of the source on large scales. Similar arguments can be made for ion temperature gradient (ITG) modes (Cowley et al., 1991), in particular also because gradients in ion temperature in space plasmas will almost always be accompanied by plasma density gradients mixing the gradient drift and the ITG modes of oscillation. The remaining shortcoming will be the omission of longitudinal velocity shear instabilities where the flow is along magnetic field lines (D'Angelo and von Goeler, 1966). This instability and its nonlinear, possibly turbulent saturated stage deserves further scrutiny.

\section{Turbulence modelling}

The ideas of turbulence (strong turbulence, in particular) were first formulated for incompressible neutral flows (Chandrasekhar, 1957; Tennekes and Lumley, 1972), and a short summary of early results obtained can be useful here, in particular also because they were closely followed by some early attempts to discuss low-frequency turbulence in plasmas.

\subsection{Models for strong turbulence in fluids and plasmas}

It is generally believed that the Navier-Stokes equation is adequate for describing all the scales that are important and relevant for modelling incompressible turbulent neutral flows. This is a relatively simple differential equation, after all, being of first order in time and second order in spatial differentials. Some of the solutions of this equation are simple as well. The solution represented by a turbulent flow has, however, so far escaped a complete understanding. Indeed, there was a time when it was expected that turbulence phenomena were to remain incomprehensible, and it probably came as a surprise that while understanding an individual turbulent flow is beyond our reach, some simple laws could be predicted for statistical averages (Frisch, 1995), the secondorder structure function in particular. Some results by can be argued by simple dimensional reasoning (Chandrasekhar, 1957).

For incompressible neutral flows the mass density does not explicitly play a part, and the kinematic viscosity $v$ accounts for the properties of the fluid. At least on small scales, the 
distribution of energy over the directions of wave numbers can be assumed to be uniform, i.e. the turbulence has become isotropic (Chandrasekhar, 1957). Concerning the turbulence, we assume that it can be adequately described by the average specific energy dissipation $\epsilon$, which for steadystate conditions is also the average energy input. It is usually assumed that the bounding box is so large that its size $\mathcal{L}$ can be taken to be irrelevant. More precisely, $\mathcal{L}$ is the scale size of the largest energy-containing eddies in the turbulence. This statement can be quantified in terms of the correlation function for a particular fluctuating quantity $\Psi(r, t)$ that can represent a velocity component or any other relevant quantity. The two-time, two-position correlation function can always be written as $R_{\Psi}\left(r_{1}, t_{1}, r_{2}, t_{2}\right)$. Spatially homogeneous turbulence requires that $R_{\Psi}$ is a function of spatial separations $r_{1}-r_{2}$ rather than $r_{1}$ and $r_{2}$ individually. We can always write $R_{\Psi}\left(r_{1}, t_{1}, r_{2}, t_{2}\right)$ as $R_{\Psi}\left(R, t_{1}, r, t_{2}\right)$ with $R \equiv \frac{1}{2}\left(r_{1}+r_{2}\right)$ and $r \equiv r_{1}-r_{2}$, i.e. $r_{1} \equiv R+\frac{1}{2} r$ and $r_{2} \equiv R-\frac{1}{2} r$. Local homogeneity implies that $R_{\Psi}\left(R, t_{1}, r, t_{2}\right)$ varies much more slowly with $R$ than with $r$. In this case we can Fourier transform $R_{\Psi}\left(R, t_{1}, r, t_{2}\right)$ with respect to the variable $r$ and thereby obtain a local power spectrum (Pécseli, 2000), referring to the position $R$. For laboratory experiments we can safely assume the turbulence to be a stationary random process, giving the correlation function $R_{\Psi}(R, r, \tau)$ with $\tau \equiv t_{1}-t_{2}$. This will not often be so for space experiments. If the Fourier spectrum with respect to a transform in $r$ contains much smaller turbulent wavelengths than the Fourier transform with respect to $R$, we can argue for a separation in scales and ignore the size of the bounding box as long as we consider only wave numbers $k \gg 1 / \mathcal{L}$. In this limit, small-scale processes can be described by universal laws, irrespective of $\mathcal{L}$.

We want first to obtain an expression for the wave number power spectrum $S(k)$ for the turbulent fluctuations in neutral fluid velocity in three spatial dimensions. The arguments and methods apply, however, independently of the dimensionality of the problem. The spectrum is here defined so that $\left\langle u^{2}\right\rangle=\int_{0}^{\infty} S(k) \mathrm{d} k$ rather than $\left\langle u^{2}\right\rangle=$ $\iiint_{-\infty}^{\infty} S(k) \mathrm{d} k_{x} \mathrm{~d} k_{y} \mathrm{~d} k_{z}$, implying here the physical dimension $[S(k)]=L^{3} T^{-2}$, with $L$ and $T$ here denoting length and time, respectively. It is known (Buckingham, 1914) that any physically meaningful function can be written as a dimensionally correct coefficient multiplied by a dimensionless function of dimensionless variables. This will always be so, but the observation need not be of much help for solving the problem. The present case is, however, simplified by noting that, given $k, v$ and $\epsilon$, with $[k]=L^{-1},[v]=L^{2} T^{-1}$ and $[\epsilon]=L^{2} T^{-3}$, only one possible combination can give a dimensionally correct velocity power spectrum

$S(k)=\left(\epsilon v^{5}\right)^{1 / 4} \mathcal{G}\left(\frac{k v^{3 / 4}}{\epsilon^{1 / 4}}\right)$,

where the dimensionless function $\mathcal{G}(x)$ of a dimensionless variable $x$ is so far undetermined. It is now a common knowl- edge that viscosity only has an affect on the very small scales in a flow, and we can expect, still provided that $\mathcal{L}$ is large enough, that an interval for $k$ can exist, where the spectrum is independent of $v$. For this to be the case, we require $v$ to cancel out (Chandrasekhar, 1957) and find here that this implies the functional form $\mathcal{G}(x) \sim x^{-5 / 3}$, giving the inertial range of the spectrum

$S(k)=C_{K} \epsilon^{2 / 3} k^{-5 / 3}$,

the well-known Kolmogorov-Oubokhov velocity power spectrum. Studies of turbulent flows in the laboratory and in nature have given the Kolmogorov-Oubokhov spectrum solid support. A universal numerical constant $C_{K}$ cannot be determined by dimensional reasoning (Sreenivasan, 1995). Experimentally, it is found that $C_{K} \approx 1.6 \pm 0.2$. The inertial subrange extends to scales comparable to the Kolmogorov length scale $\eta=\left(v^{3} / \epsilon\right)^{1 / 4}$.

We have the relation $\epsilon=2 v \int_{0}^{\infty} k^{2} S(k) \mathrm{d} k$, where the integral covers the entire spectrum (Batchelor, 1953). The $k^{2}$ multiplier in the integrand ensures that the contribution from the large non-universal scales is suppressed. For $k>$ $1 / \eta$ we find the dissipation subrange, which in the classical Heisenberg analysis (Heisenberg, 1948; Chandrasekhar, 1949) is found to decrease like $k^{-7}$. This particular results seems to agree well with some observations but is nonetheless suspect since it predicts that higher-order velocity derivatives $\left\langle\left(\nabla^{j} u\right)^{2}\right\rangle=\int_{0}^{\infty} k^{2 j} S(k) \mathrm{d} k$ should be divergent for some large $j$. There are no indications of the NavierStokes equation having this property (Sulem et al., 1983; Constantin, 1991). Models containing power law spectra extending to infinity (in wave number or frequency) should be considered with suspicion.

The foregoing arguments emphasized spectra in terms of wave vectors. It is easily demonstrated that similar arguments can be used to find an inertial subrange for frequency spectra, giving

$S(\omega)=C_{F} \epsilon \omega^{-2}$,

with $C_{F}$ being a universal constant. It is unfortunately not obvious how Eq. (3) should be interpreted. For the wave number spectrum Eq. (2), we Fourier transform a spatially varying correlation function at any given time, and no ambiguity is found. A temporal record can, however, be obtained by, for instance, a fixed observer (Eulerian sampling) or by an observer moving passively with the fluid (Lagrangian sampling). The velocity field might be sampled along a curved prescribed trajectory as well. Only the Eulerian sampling has practical relevance here. The Eulerian frequency spectrum will, however, contain contributions from large non-universal scales with size $\mathcal{L}$ sweeping small-scale turbulences past the observer (Tennekes, 1975). These large scales will be the most energetic and their non-universal contributions will dominate any universal part of the frequency spectrum. Most analytical studies are concerned with wave number spectra, where the interpretation is unambiguous. 
When it was realized that plasmas can also develop a turbulent state, it was argued that the dimensional arguments found for fluid turbulence might be applied also to the plasma case and electrostatic drift wave turbulence in particular (Chen, 1965a). Dimensional arguments can be used irrespective of the dimensionality of the problem and the foregoing discussions can be applicable also for the plasma problem. It was argued (Chen, 1965a) that five dimensional parameters suffice to account for the basic properties of drift wave turbulence with cold ions: the ion mass $M$, the elementary charge $e$, the sound speed $C_{\mathrm{s}}=\sqrt{T_{\mathrm{e}} / M}$ in terms of the electron temperature $T_{\mathrm{e}}$; the effects of the magnetic field are introduced via the ion gyro frequency $\Omega_{\mathrm{ci}}=e B / M$. These parameters are independent as none of them can be constructed through a combination of the others. A characteristic length scale, the effective ion Larmor radius, can be constructed as $a_{i} \equiv C_{\mathrm{s}} / \Omega_{\mathrm{ci}}$, which estimates the shortest wavelength for drift waves. The power spectrum of the electrostatic potential is introduced as $\left\langle\widetilde{\phi}^{2}\right\rangle=\int_{0}^{\infty} G_{C}(k) \mathrm{d} k$, having the dimension (mass $\times$ length ${ }^{2} \times$ charge $^{-1} \times$ time $\left.^{-2}\right)^{2}$, giving $\left[G_{C}(k)\right]=(M / e)^{2} L^{5} T^{-4}$. If we were to discuss the power spectrum of relative density variations, giving a dimensionless quantity $\left\langle\left(\widetilde{n} / n_{0}\right)^{2}\right\rangle$, the arguments could easily be generalized to this problem as well. With the foregoing arguments we find that with the given parameters, the only dimensionally correct combination is

$G_{C}(k)=\left(\frac{M}{e}\right)^{2} \Omega_{\mathrm{ci}}^{4} a_{i}^{5} \mathcal{F}\left(k a_{i}\right)$.

If we are to have a universal long-wavelength range independent of $a_{i}$, we require $\mathcal{F}(x) \sim x^{-5}$ for a particular subrange for $a_{i}$ to vanish from Eq. (5). For drift waves within this model, we then have

$G_{C}(k) \propto\left(\frac{M}{e}\right)^{2} \Omega_{\mathrm{ci}}^{4} k^{-5}$.

However, this result cannot possibly be correct, since it predicts a turbulent spectrum independent of any energy input, i.e. also in the absence of a density gradient (i.e. no drift waves), for instance. The shortcoming is due to an oversimplification of the parameters needed to account for the basic properties of drift wave turbulence. Although the result is in error, the paper by Chen (1965a) was nonetheless a pioneering work as it argued that ideas from strong neutral turbulence could be generalized to plasmas and reasoned that universal laws may be found also for plasma turbulence. A different derivation by Fowler (1966) of the $k^{-3}$ power spectrum for electric field fluctuations tried in part to remedy the shortcomings of Eq. (5) by introducing the macroscopic radius of the plasma column as an outer scale, but the result remained independent of the nature of the density gradient.
Other analytical works (Tchen et al., 1980) found a characteristic frequency for drift wave turbulence,

$\omega_{0} \equiv\left(\Omega_{\mathrm{ci}} \int_{0}^{\infty} k^{2} G_{T}(k) \mathrm{d} k\right)^{1 / 3}$,

which controls what can be called the equivalent of an inertial spectral range, thus in a sense replacing $\Omega_{\mathrm{ci}}$ from the first attempt Eq. (5) to derive a universal spectral law for drift wave turbulence. The $k^{2}$ multiplier in the integrand of $\omega_{0}$ ensures that the contribution to $G_{T}(k)$ from the large non-universal scales is suppressed. For the analysis it turned out to be an advantage to introduce $\psi \equiv \sqrt{\left(T_{\mathrm{e}}+T_{i}\right) / M} \ln \left(n / n_{\mathrm{r}}\right) \equiv$ $C_{\mathrm{S}} \ln \left(n / n_{\mathrm{r}}\right)$, assuming isothermal ions, while $n_{\mathrm{r}}$ is a fixed normalizing reference density and $G_{T}(k)$ entering Eq. (6) is the power spectrum of $\psi$. With this modified frequency $\omega_{0}$, it is possible to recover the $k^{-5}$ spectrum for the normalized electrostatic potential, where the spectrum is defined so that $\left\langle\psi^{2}\right\rangle=\int_{0}^{\infty} G_{T}(k) \mathrm{d} k$. The physical dimensions for $k G_{T}(k)$ are here length ${ }^{2} \times \operatorname{time}^{-2}$, giving $\left[G_{T}(k)\right]=L^{3} T^{-2}$. The drift wave power spectrum was found to be

$G_{T}(k) \sim \frac{\omega_{0}^{4}}{\Lambda_{c} \Omega_{\mathrm{ci}}} k^{-5}$,

where $\Lambda_{c} \equiv a_{D}\left(T_{\mathrm{e}} / M\right) / \Omega_{\mathrm{ci}}$ enters the analysis with an undetermined numerical coefficient $a_{D}$. Note that $\Lambda_{c}$ has the dimension length ${ }^{2} \times$ time $^{-1}$. For low levels of $G_{T}(k)$, i.e. large $k$, we have $\widetilde{\psi} \approx C_{\mathrm{s}} \tilde{n} / n_{0}$ for the fluctuating quantities, so that Eq. (7) gives the density spectrum in that limit. The analysis assumes nearly Boltzmann-distributed electrons so that $\widetilde{n} \sim \widetilde{\phi}$ for large $k$, so that the spectral indexes for density and potential fluctuations are similar. The steady-state density gradient need not have any potential variation associated with it. The isothermal electron Boltzmann equilibrium relates only to perturbations of the initial density variation. Possible initial large-scale steady-state electric fields have their own sources.

The result Eq. (7) together with Eq. (6) can be seen as a self-consistency relation for the power spectrum just as for the Kolmogorov-Oubokhov spectrum (there with $\epsilon=$ $\left.2 v \int_{0}^{\infty} k^{2} S(k) \mathrm{d} k\right)$. Different subranges of the turbulent spectrum were identified (Tchen et al., 1980), in particular also a long-wavelength spectral part, i.e. a production subrange for the power spectrum of the electrostatic potential

$G_{T}(k) \sim \Gamma_{\psi}^{2} k^{-3}$,

with $\Gamma_{\psi} \equiv C_{\mathrm{s}} n_{0}^{-1}\left|\nabla n_{0}\right|$ characterizing the inverse density gradient length scale. This limiting spectral subrange has a counterpart in neutral velocity shear-driven turbulence (Tchen, 1953; Tchen and Pierson, 1988). 
A transition wave number separating the production and coupling subranges (i.e. the $k^{-3}$ and $k^{-5}$ subranges) can be found as

$k_{c}=\frac{\omega_{0}^{2}}{\sqrt{\Lambda_{c} \Omega_{\mathrm{ci}}}} \frac{1}{\left|\Gamma_{\psi}\right|}$,

where $\Lambda_{c}$ contains a numerical constant which is not well known. $k_{c}$ is easily identified in the power spectra for density and potential fluctuations. Its parameter variation can be studied experimentally (Pécseli et al., 1983) and also by numerical simulations, but only little work has been done on this problem. The wave number range of the spectrum given by Eq. (7) is limited by $k_{c}$ on large scales and by $k \sim 1 / a_{i}$ on small scales. The work by Tchen et al. (1980) includes a short-wavelength limit, but it is also pointed out that this subrange may not develop. The contribution of this subrange to the spectral integral in $\omega_{0}$ will be negligible anyhow.

\subsection{Strong- versus weak-turbulence models}

It is by no means obvious that results from strong-turbulence studies apply to plasmas as well (Dupree, 1969), at least not in general. To discuss possible limitations in the analysis, we first rewrite the Navier-Stokes equation after a Fourier transform as

$$
\begin{aligned}
& \frac{\partial}{\partial t} u_{i}(k, t)+v k^{2} u_{i}(k, t)= \\
& \sum_{j, \ell ; p} M_{(i, j, \ell)} u_{j}(p, t) u_{\ell}(k-p, t),
\end{aligned}
$$

where the linear viscous second term on the left side is negligible in the inertial subrange. The relative magnitude of the nonlinear coupling coefficient on the right-hand side is measured by the Reynolds number, which can be written as the ratio of the order of magnitude of the nonlinear term and the linear term

$\operatorname{Re}=\frac{\left|M_{(i, j, \ell)} u^{2}\right|}{k^{2} v u}=\frac{\left|M_{(i, j, \ell)} u\right|}{k^{2} v}=\frac{u}{k v}=\frac{\mathcal{L} u}{v}$.

Recalling the dimension $\left[M_{(i, j, \ell)}\right]=$ length $^{-1}$, we assumed that $M_{(i, j, \ell)}$ is of the order of $k$, which can be demonstrated by a calculation of the coupling coefficients and then letting the smallest wave number relevant be $k \sim 1 / \mathcal{L}$, where $\mathcal{L}$ is a characteristic system size (Dupree, 1969). The Reynolds number can also be seen as the ratio between two timescales, the damping time $k^{2} v$ and a nonlinear coupling time $\left|M_{(i, j, \ell)} u\right|$. The Reynolds number obtained by these arguments generally depends on the wave number $k$ considered: in the original formulation by Reynolds, $\mathcal{L} \sim 1 / k$ was taken to be the diameter of a tube, for instance, i.e. a fixed length scale. Strong turbulence in fluids is found when $\operatorname{Re} \gg 1$, implying that the nonlinear term on the right-hand side of Eq. (10) completely dominates the second linear term on the left side.
Within fluid models a class of nonlinear equations for plasma dynamics can be written (Dupree, 1969; Diamond et al., 2010) in the form

$$
\begin{aligned}
& \frac{\partial}{\partial t} E_{i}(k, t)+i \omega(k) E_{i}(k, t)= \\
& \sum_{j, \ell ; p} Q_{(i, j, \ell)} E_{j}(p, t) E_{\ell}(k-p, t),
\end{aligned}
$$

now expressed in terms of electric field components to make it distinct from Eq. (10), allowing also for compressible motions. The summation can include restrictions on wave vector triplet interactions. The dispersion relation derived from linear theory is here $\omega=\omega(k)$. The model equation Eq. (11) can for instance describe interactions of waves due to a quadratic nonlinearity where the coupling coefficients are $Q_{(i, j, \ell)}$. In many cases the second term on the left side of Eq. (11) will be large for all wave numbers. Often there is only a modest amount of free energy available for instabilities (Dupree, 1969) so that $E$ will not be large either. An effective Reynolds number $\left|Q_{(i, j, \ell)} E\right| / \omega(k)$ will therefore often be of the order unity for a wide band of wave numbers, and a strongly turbulent state is unlikely to develop in the sense described for neutral fluids (Dupree, 1969). In some cases the linear and nonlinear terms balance exactly, and we can find solitary or even soliton solutions that possess an inverse scattering transform (Drazin and Johnson, 1989). Strong turbulence is found for cases where the linear dispersion relation is immaterial, and possible restrictions on triplet interactions are relaxed.

In many studies of seemingly turbulent plasmas it is possible, by suitable chosen multiprobe diagnostic methods (Iwama et al., 1979; Beall et al., 1982), to determine a relation between frequency and wave vector experimentally. An illustrative example is shown in Fig. 1. Although these experimental relations are subject to some broadening and therefore not dispersion relations in a traditional sense, their presence indicates that the $i \omega(k) E_{i}(k, t)$ term in Eq. (11) is not negligible in comparison to the righthand side. Consequently the plasma conditions are not properly be called "strongly turbulent". The dispersion relations mentioned here differ from the frequency-wave-number proportionality implied by the use of the Taylor hypothesis (Shkarofsky, 1969; Hinze, 1975), or the "frozen turbulence approximation" to be discussed later.

It turns out that drift waves offer one example of plasma waves where a strongly turbulent state may develop. A close analogy can be found, for instance, with waveforms in the Earth's neutral atmosphere, the Rossby waves. A simple model equation was derived by Hasegawa and Mima (1978). Introducing the potential vorticity $q \equiv \phi-\nabla_{\perp}^{2} \phi-\vartheta x$ the Hasegawa-Mima equation can be expressed as an Euler equation

$$
\left(\frac{\partial}{\partial t}+\boldsymbol{U} \cdot \nabla_{\perp}\right) q=0
$$




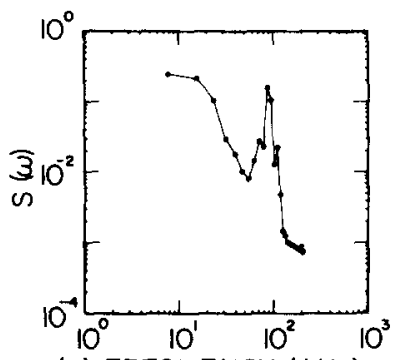

(a) FREQUENCY $(\mathrm{kHz})$
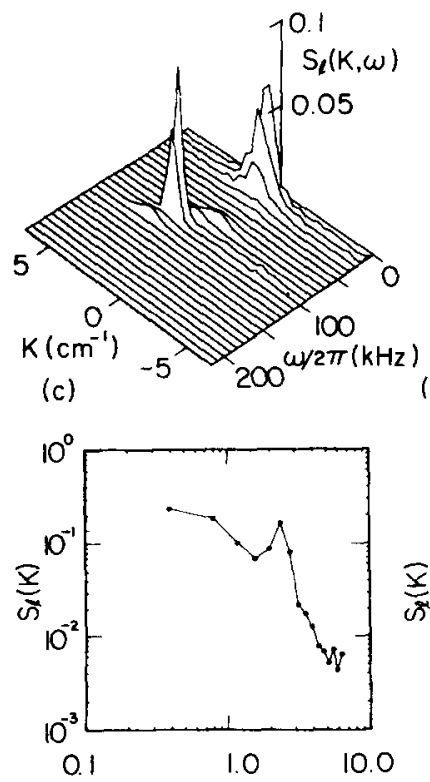

(e) WAVE NUMBER $\left(\mathrm{cm}^{-1}\right)$

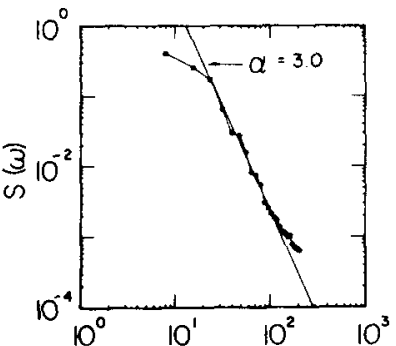

(b) FREQUENCY $(\mathrm{kHz})$
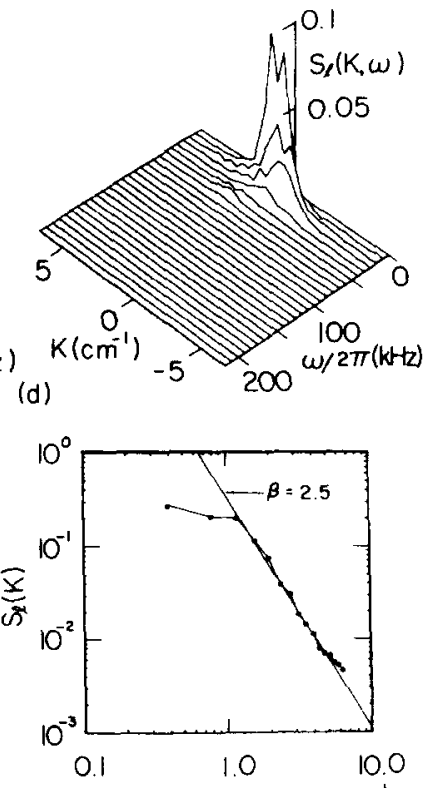

(f) WAVE NUMBER $\left(\mathrm{cm}^{-1}\right)$
Figure 1. Summary figure from experimental studies of plasma density fluctuations in radio frequency (RF) discharges, obtaining dispersion relations against a noisy background (Iwama et al., 1979; Beall et al., 1982). For the conditions on the left it is feasible to obtain evidence of a dispersion relation. For the fully developed turbulent conditions on the right-hand side, all that remains is a "blur" for small $\omega$ and $k$. Reproduced with permission from Beall et al. (1982). Copyright 1982, AIP Publishing LLC.

where $\phi$ is the electrostatic potential, $\boldsymbol{U} \equiv-\nabla_{\perp} \phi \times \boldsymbol{B} / B^{2}$ is the lowest-order guiding centre velocity and $\vartheta \equiv$ $n_{0}^{\prime}(x) / n_{0}(x)$ measures the density gradient. For small $\vartheta$ the relation Eq. (12) is inherently nonlinear, just like Eq. (10) in the limit of small $v k^{2}$, although we note that the Fourier transform of $q$ exists only in a limiting sense.

The Hasegawa and Mima equation accounts for the nonlinear coupling of modes, but describes linearly stable plasma, with no energy input or dissipation. An extended and generalized model of the Hasegawa and Mima equation (Hasegawa and Wakatani, 1983) produces linearly unstable conditions, with dissipation models included ad hoc. For resistive electrostatic drift waves with electron-ion collision frequency $\nu_{\mathrm{ei}} \equiv \tau_{\mathrm{ei}}^{-1}$, we have the complex dispersion relation (Chen, 1965d, e; Weiland, 2000; Pécseli, 2012) for linear waves,

$\omega\left(\omega-\omega_{i}\right)+i \sigma_{\|}\left[\omega-\omega^{*}+b\left(\omega-\omega_{i}\right)\right]=0$,

using the seemingly universal notation $\sigma_{\|} \equiv$ $\left(k_{z}^{2} / k_{y}^{2}\right)\left(\omega_{\mathrm{ce}} \tau_{\mathrm{ei}}\right) \Omega_{\mathrm{ci}}, \quad b \equiv k_{y}^{2} a_{i}^{2}$ with $a_{i}^{2} \equiv T_{\mathrm{e}} /\left(M \Omega_{\mathrm{ci}}^{2}\right)$ and the drift frequency $\omega^{*}=k_{y} U_{\mathrm{De}}=-k_{y}\left(n_{0}^{\prime} / n_{0}\right) T_{\mathrm{e}} /(e B)$, while $\omega_{i} \equiv-\theta \omega^{*}$ with $\theta \equiv T_{i} / T_{\mathrm{e}}$ and while $n_{0}=n_{0}(x)$ is the unperturbed plasma density with $\hat{\boldsymbol{x}} \perp \boldsymbol{B}$. The ambient homogeneous magnetic field $\boldsymbol{B}$ is in the $z$ direction. $U_{\mathrm{De}}$ is the electron diamagnetic drift velocity. Damped waves propagate with the ion diamagnetic drift velocity. The basic properties of the dispersion relation Eq. (13) can be illustrated by the simplified results for cold ions, $T_{i} \approx 0$; see Fig, 2. The linear instability of the resistive drift waves is caused by a small phase difference between the potential and the electron density due to the electron-ion collisions that prohibit the free electron flow along magnetic field lines from wave crest to wave trough; see Fig, 2c. The isothermal Boltzmann equilibrium of the electrons is thereby perturbed. Collisions between charged particles and neutrals have the same effect, with one significant difference: while electronion collisional diffusion along a density gradient will not give rise to any steady-state electric fields, the difference in electron and ion mobilities due to neutral collisions will imply the build up of an electric field in the latter case (Pécseli, 2012). This electric field will have a magnitude of the order of $(T / e)\left|\nabla \ln n_{0}\right|$, with $T$ being a characteristic plasma temperature. Since the mobility of the ions across magnetic field lines is much larger than for the electrons, their motion will induce an electric field with a direction being the same as the plasma density gradient direction. In the ionosphere we can have additional steady-state electric fields imposed by, for instance, the interaction between the solar magnetic field and the Earth's magnetosphere (Kelley, 1989).

These models, and those derived from them, formed the starting points of many studies (although not all) of strong drift wave turbulence. Investigations of turbulent drift waves have been stimulated by their importance for anomalous transport in magnetized plasmas and fusion plasma experiments in particular (Liewer, 1985; Wootton et al., 1990; Misguich et al., 1987; Horton, 1990, 1999). The present summary implicitly assumes locally homogeneous magnetic fields, but drift wave turbulence has been observed in more complex magnetic field configurations such as stellarators (Bol, 1964; Birkenmeier et al., 2013).

None of the early analytical studies of drift wave turbulence (Chen, 1965a; Fowler, 1966; Tchen et al., 1980) distinguished energy and enstrophy cascades (Kraichnan, 1967, 1971; Chekhlov et al., 1996). When energy is injected on a particular intermediate length scale for turbulence in two spatial dimensions, say at $\lambda_{0}$, energy can be assumed to cascade towards scales larger than $\lambda_{0}$, with enstrophy (i.e. the square of the rotation of the velocity field) cascading to scales smaller than $\lambda_{0}$. The two subranges $\lambda<\lambda_{0}$ and $\lambda>\lambda_{0}$ have 


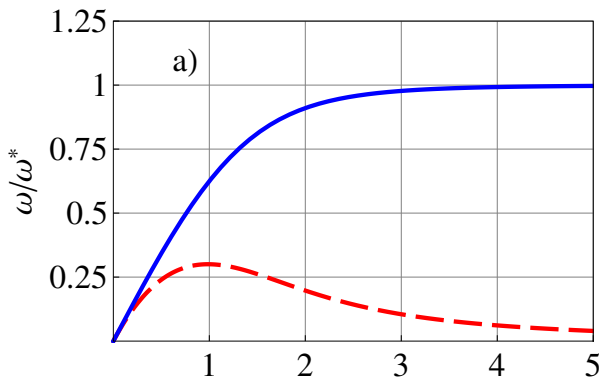

$\left(k / k_{y}\right) \sqrt{\omega_{c} \tau_{\mathrm{ei}} \Omega_{c} / \omega^{*}}$
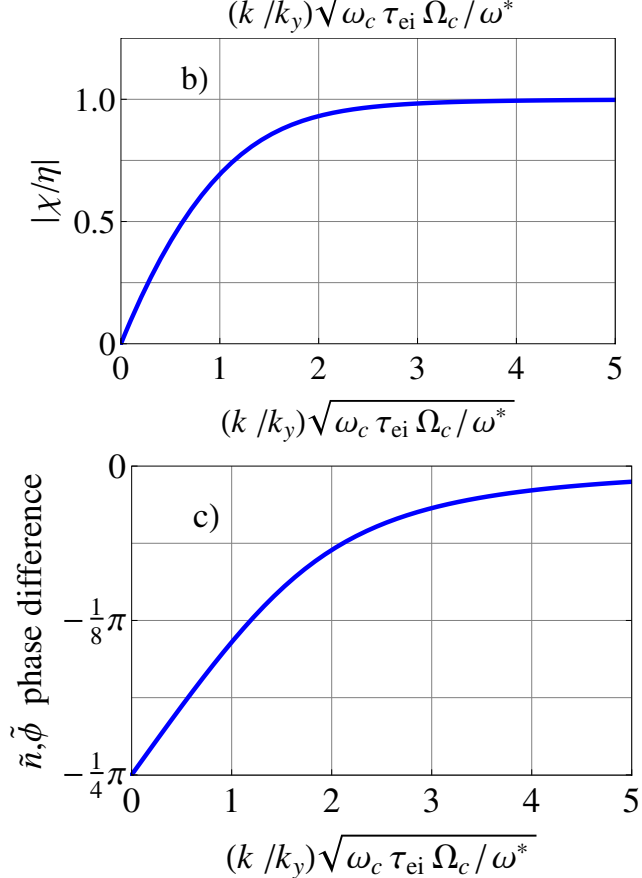

Figure 2. Summary of the normalized dispersion relation Eq. (13) shown in (a), with full line giving the real part of the frequency and dashed line the normalized growth rate. For the purposes of illustration, we here took $T_{i}=0$ and $b=0$, thereby ignoring ion polarization drifts. In (b) we show the ratio between normalized electrostatic potential $\chi \equiv e \widetilde{\phi} / T_{\mathrm{e}}$ and normalized density $\eta \equiv \widetilde{n} / n_{0}$, while (c) gives the phase difference between fluctuating plasma density and potential for linear drift waves (Pécseli, 2012).

different spectral laws. The enstrophy cascade is a feature of some two-dimensional systems and applies to neutral flows as well as low-frequency turbulence in magnetized plasmas. The concept is supported by numerical simulations (Lilly, 1969). The present review primarily addresses observations in laboratory and space plasmas, and the enstrophy cascade in the form outlined above has found limited support there. An experiment was carried out by Huld et al. (1988), where energy was injected on a small localized scale at a particular initial time. The subsequent temporal evolution of the spatial energy distribution as given by the inhomogeneous, nonstationary correlation function was then followed. Details of the energy cascade could, in principle, be distinguished by this a)

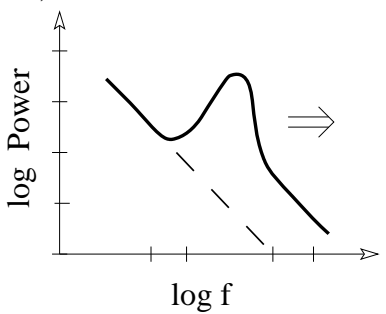

b)

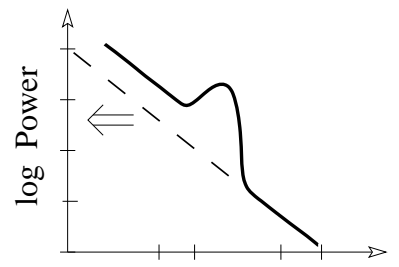

$\log \mathrm{f}$
Figure 3. Schematic illustration of spectra observed by Roth (1971) in a modified Penning discharge, with a "common sense" argument for the direction of a spectral cascade given by enhancement in power levels as indicated by double arrows. We have energy cascade to shorter wavelengths in (a) and the inverse cascade in (b).

procedure. The results were not conclusive. Simple and elegant arguments for identifying the direction of the energy cascade have been suggested by Roth (1971) and described in detail there; see also Fig. 3. The ideas can apply to cases where a peak is found in the spectrum, as in the left side of Fig. 1. In most cases where such features are observed (Roth, 1971; Smith and Powers, 1973), the arguments seem to favour models where energy cascades to large frequencies and large wave numbers. Experiments on soap films (this comes as close to truly two-dimensional systems as is possible) have given support for the dual energy-enstrophy cascade (Rutgers, 1998).

\subsection{Universal spectra in a weak-turbulence model}

Universal spectral laws can be derived also within a weakturbulence theory. One of these results seems particularly relevant here. The current generated ion acoustic instability (i.e. an instability generated by an electron flow with velocity $u$ relative to the ions) saturates at a moderate level of turbulent electrostatic fluctuations, and analytically it was found (Kadomtsev, 1965) that the power spectrum of the normalized electrostatic potential for $u \gg C_{\mathrm{s}}$ becomes

$I_{k} \equiv\left\langle\left(\frac{e \phi(k)}{T_{\mathrm{e}}}\right)^{2}\right\rangle \sim \frac{u}{u_{\mathrm{th}, \mathrm{e}}} \frac{T_{\mathrm{e}}}{T_{i}} \frac{1}{7 \theta_{0}^{2}} \frac{1}{4 \pi k^{3}} \ln \left(\frac{1}{k \lambda_{D}}\right)$

for $k \lambda_{D}<1$ with $u_{\text {th,e }} \equiv \sqrt{2 T_{\mathrm{e}} / m}$. The potential power spectrum is here defined so that $\left\langle\left(e \phi / T_{\mathrm{e}}\right)^{2}\right\rangle=\iiint_{\mathcal{V}} I_{k} \mathrm{~d}^{3} k$, with the integration taken over a cone of wave vector directions with an opening angle $\theta_{0}$ in three-dimensional wave vector space. For $k \ll 1 / \lambda_{D}$ we find $I_{k} \sim k^{-3}$ to be a good approximation. For the electric field power spectrum, we have $E^{2}(k)=k^{2} I_{k}$. The cone angle $\theta_{0}$ of propagation directions is not well defined. The resulting Eq. (14) has received some experimental support (Machalek and Nielsen, 1973), although this experiment measured density fluctuations, and it is not evident what the relation between density and potential fluctuations is for a turbulent-current-carrying plasma. It 
is interesting that the logarithmic correction in Eq. (14) was also included in the experimental fit (Machalek and Nielsen, 1973) with good agreement. The experiment was carried out at a hydrogen plasma density of $n \approx 4 \times 10^{20} \mathrm{~m}^{-3}$, with $T_{\mathrm{e}} \approx T_{i} \approx 1 \mathrm{eV}$ in a $\boldsymbol{B}$-perpendicular shock at initially $B \in$ $\{0.035-0.1\} \mathrm{T}$. Mach numbers were in the range $\{1.5-6\}$. Observed fluctuation frequencies were below the ion plasma frequency, following a measured sound-like dispersion relation with a phase velocity comparable to the ion thermal velocity, which is close to the ion sound speed for the present parameters. The plasma conditions are thus not those used for deriving Eq. (14), but the free energy for the turbulence can nonetheless be identified as an electron current, in this case the current perpendicular to $\boldsymbol{B}$. It was not attempted to identify an instability mechanism in the experiment.

The fluctuation level obtained from Eq. (14) can become large for small $k$. The theoretical model has the basic assumption that the current is due to a displaced Maxwellian electron velocity distribution. For a high fluctuation level this model for the electron velocity distribution may not be appropriate. The lowest-order modifications taking distortions of the velocity distribution into account (Kadomtsev, 1965) did not alter the $k^{-3}$ dependence in Eq. (14).

\subsection{Taylor's hypothesis}

Experiments in the laboratory and in space obtain frequency spectra which are subsequently interpreted as wave number spectra with reference to the Taylor hypothesis (or the frozen turbulence approximation) often used in studies of fluid and plasma turbulence (Shkarofsky, 1969). The Taylor hypothesis basically argues for the replacement $\boldsymbol{r} \rightarrow \boldsymbol{U} t$ in the detected correlation functions $R_{\mathrm{c}}$, stating that $R_{\mathrm{c}}(r=0, t)$ $\approx R_{\mathrm{c}}(r=\boldsymbol{U} t, t=0)$, or the equivalent $\omega \rightarrow \boldsymbol{U} \cdot \boldsymbol{k}$ replacement in the detected power spectra, where $\boldsymbol{U}$ is the detector velocity (Shkarofsky, 1969). A detected frequency in a power spectrum is interpreted in terms of the Doppler shift, $\omega \approx \boldsymbol{k} \cdot \boldsymbol{U}$, for the case where the plasma is moving with a high velocity $\boldsymbol{U}$ past a detector, the tip of a Langmuir probe for instance. The hypothesis has to be supplemented by assumptions about the distribution over the wave vector directions (Fredricks and Coroniti, 1976), hypothesizing, for instance, local isotropy in a plane perpendicular to the magnetic field. For low observed frequencies it will generally not be correct to assume that the Doppler shift dominates. Limitations in the applicability of the Taylor hypothesis were discussed by Shkarofsky (1969). No universal criterion can be given for the applicability of the hypothesis, but some reasonably general models can be postulated stating the requirements to be $U^{2} \gg\left\langle\widetilde{u}^{2}\right\rangle$ and $t^{2} k_{0}^{2}\left\langle\widetilde{u}^{2}\right\rangle \ll 1$ when applied to the autocorrelation function. The application of the hypothesis for power spectra assumes $U^{2} \gg\left\langle\widetilde{u}^{2}\right\rangle$ and $\omega^{2} \gg k_{0}^{2}\left\langle\widetilde{u}^{2}\right\rangle$. In both cases we have $\widetilde{u}$ as the fluctuating velocity in the medium, either fluid or plasma, and $k_{0}$ representing the inverse of the scale size of the largest energy-containing eddies
(Shkarofsky, 1969). In practice it is often found that the Taylor hypothesis can be applied with relatively mild restrictions (Tennekes, 1975).

For one-dimensional turbulence (should it exist), the relations between the observed power spectrum $S_{0}(\omega=\boldsymbol{k} \cdot \boldsymbol{U})$ of a particular fluctuating scalar quantity and the actual power spectrum $S(k)$ are trivial. For a two-dimensional case we have $S_{0}\left(k_{x}\right)=\int_{-\infty}^{\infty} S\left(k_{x}, k_{y}\right) \mathrm{d} k_{y}$, while in three dimensions we have $S_{0}\left(k_{x}\right)=\iint_{-\infty}^{\infty} S\left(k_{x}, k_{y}, k_{z}\right) \mathrm{d} k_{y} \mathrm{~d} k_{z}$, with the $\hat{x}$ axis taken along $\boldsymbol{U}$. If, in addition, we can assume isotropy in three dimensions, $S(k)=S(|k|)$, so that $S\left(k_{x}, k_{y}, k_{z}\right) \mathrm{d} k_{y} \mathrm{~d} k_{z}=S\left(\sqrt{k_{x}^{2}+k_{y}^{2}+k_{z}^{2}}\right) \mathrm{d} k_{y} \mathrm{~d} k_{z} \rightarrow$ $2 \pi S\left(\sqrt{k^{2}+k_{x}^{2}}\right) k \mathrm{~d} k$, we have the relation

$S(k)=-\frac{1}{2 \pi k} \frac{d S_{o}(k)}{\mathrm{d} k}$,

without requirements for a power law spectrum. For two spatial dimensions no such simple relation exists. The advantage of Taylor's hypothesis is that it requires only one probe for detection. More advanced methods that can estimate wave number spectra directly require two or more probes (Smith and Powers, 1973; Iwama et al., 1979; Beall et al., 1982).

It might be instructive to show the result for a case where the turbulence is confined to a part of wave number space: assume we have $S(k)=A|k|^{-\alpha}$ within a cone with an opening angle $\theta_{0}$ as in Eq. (14) and $S(k)=0$ outside. For $k \sec \psi_{0} \tan \theta_{0}>0$, we find, after some algebra,

$$
\begin{aligned}
S_{0}(k)= & A k^{2-\alpha} 2^{-\alpha / 2} \pi \\
& \times \sec ^{2}\left(\psi_{0}\right) \tan \left(\theta_{0}\right) \sin \left(\theta_{0}\right) \sec \left(\theta_{0}-\psi_{0}\right) \\
& \times{ }_{2} \mathrm{~F} 1\left[\frac{1}{2}, \frac{\alpha}{2}, 2,-\frac{1}{2} \sec ^{2} \psi_{0} \tan ^{2} \theta_{0}\right],
\end{aligned}
$$

where $\psi_{0}$ is the angle between the detector trajectory and the cone axis and ${ }_{2} \mathrm{~F} 1[a, b, c, z]$ is the hypergeometric function. A detector moving at a high speed where Taylor's hypothesis applies will thus detect a $k^{2-\alpha}$ spectrum in this case, so that a $k^{-3}$ power law in the three-dimensional space will be aliased to $k^{-1}$ by a moving detector.

For three-dimensional fluid turbulence, we have an isotropic velocity power spectrum $\sim k^{-11 / 3}$ in wave vector space. This spectrum is aliased to $\sim k^{-5 / 3}$ by a detector moving at a high speed.

Taylor's hypothesis is not restricted to power law spectra, although this form is often implied. Since these forms for spectra are found so often, they are also emphasized in this review. It should be noted, however, that arguments have been offered also for other spectral laws (Maggs and Morales, 2012), exponential spectra, for instance. It was argued by the authors that due to experimental uncertainties, it can be difficult to distinguish such spectra from those obtained by measurements. The most common source of uncertainties can be found in limited record lengths. Many laboratory experiments are, however, in a position to provide very 
long records, and this uncertainty can be strongly reduced. There is, on the other hand, no a priori guarantee that any physically acceptable random process necessarily develops power law spectra. It is, for instance, unlikely that the frequency spectrum of noise from rain falling on a tin roof will follow such a power law. It is also well known that by properly prepared synthetic time series, it is possible to generate data sets with any preselected integrable frequency power spectrum as well as bispectrum (Pécseli and Trulsen, 1993; Pécseli, 2000). A similar conclusion will be valid also for wave vector spectra in one, two or three spatial dimensions (Lynov et al., 1991).

\section{Laboratory studies}

A number of laboratory experiments have been carried out for studying drift wave turbulence (Tynan et al., 2009). Some investigations of turbulent spectra, in particular, were compiled and summarized in early studies (Chen, 1965a; Tchen et al., 1980). Thus, even before analytical results for possibly universal spectra were found, a number of experiments studied spectral distributions of fluctuations in density, potential, etc. More detailed measurements were carried out later. A summary is presented in Table 1 of this paper.

For cold plasmas, as in Q machines (Motley, 1975), measurements by Langmuir probes can provide data for variations in plasma density and potential. For hot plasmas, remote techniques, microwave scattering for instance (Okabayashi and Arunasalam, 1977), are often used.

Studies of fully developed turbulent limits with power law wave number spectra (Beall et al., 1982) found no signs of a dispersion relation, which was, on the other hand, clear for other cases. Referring to the discussion in Sect. 2.2, we take this as an indication of a strongly turbulent state being developed in the former case. The waves were interpreted as drift waves by Beall et al. (1982), but for this particular case the spectral index was close to 3 . The tokamak results shown in Table 1 seem to fall systematically below the expected spectral index for drift waves: this may be related to the distinct deviations from near isothermal Boltzmann distributions for the electrons found experimentally (Ritz et al., 1987). RF (radio frequency) discharge plasmas, on the other hand, seem in some cases to support the drift wave power spectrum (Smith and Powers, 1973) and in other cases to contradict them (Beall et al., 1982). Variations in the neutral pressures can be the cause of this discrepancy, as discussed later in Sect. 3.2. Data from a "bumpy torus" (Roth et al., 1981) show power spectra for the potential with index $\alpha \approx 4.7$, while the density has $\alpha \approx 2.8$, again in disagreement with the expected results assuming nearly Boltzmann-distributed electrons, which would predict $e \widetilde{\phi} / T_{\mathrm{e}} \approx \widetilde{n} / n_{0}$. The inhomogeneous magnetic field might be a cause of these deviations. Turbulence in a cusped magnetic field has been studied as well by D'Angelo et al. (1974): here the power spectrum of turbulent fluctuations in plasma density varied significantly from the cusp region, with weak magnetic fields, to the locally homogeneous magnetic field conditions near the magnetizing coils. In the former case an $f^{-3}$ power law was found, in the latter $f^{-5}$, which, incidentally, seems consistent with drift wave turbulence.

\subsection{Resistive drift waves with ion-electron collisions}

Systematic studies were carried out in a rotating caesium plasma column in a linear Q device (D'Angelo et al., 1974; Mikkelsen and Pécseli, 1978; Pécseli, 1982; Pécseli et al., 1983), as illustrated in Fig. 4. The high rotation velocity of the plasma allows Taylor's hypothesis to be used in the region near the radius of the plasma column. Some basic features of this experiment are summarized in Fig. 5. The fluctuation level maximizes near the position where the steady-state radial density variation is at a maximum. The relative fluctuation level $\sqrt{\left\langle(\widetilde{n} / \bar{n}(r))^{2}\right\rangle}$ stays approximately constant (within the uncertainty) for a large radial range. The parameter variations in the various spectral subranges and their "crossover" wave numbers were analysed. A sample of power spectra for density and potential fluctuations are shown in Fig. 6. The lower limit for applying Taylor's hypothesis (Shkarofsky, 1969) was estimated to be in the range of $3-5 \mathrm{kHz}$ (Mikkelsen and Pécseli, 1978). For small amplitudes, i.e. at large and intermediate frequencies, we note a close similarity of the two spectra, indicating a near proportionality between $\widetilde{n}$ and $\widetilde{\phi}$. For large amplitudes (small frequencies, large wavelengths), this similarity is no longer pronounced. Similar observations were made in studies of fluctuations in a large cylindrical magnetized plasma (Nagashima et al., 2008) and in tokamak devices (Levinson et al., 1984). The similarity of density and potential spectra at moderate fluctuation levels agrees with models where the electrons are near an isothermal Boltzmann equilibrium $e \widetilde{\phi} / T_{\mathrm{e}} \approx \ln \left(\left(\widetilde{n}+n_{0}(r)\right) / n_{0}(r)\right)$, although this relation cannot be exact for linearly unstable conditions; see Fig. 2c.

In some cases it has been possible to determine both wave number and frequency spectra simultaneously and thus test the accuracy of the Taylor hypothesis (Beall et al., 1982). Such a case is shown on the right-hand side of Fig. 1. The hypothesis is reasonably well satisfied for this case, although the relation is by no means exact. The result can be seen as an illustration of the uncertainty on the estimate of the spectral index for wave number spectra obtained through the Taylor hypothesis. Similar results were reported from measurements in a tokamak (Levinson et al., 1984), where it was noted that the wave number spectra seemed to be systematically slightly steeper than the frequency spectra. The authors mention that both density and potential spectra seemed to steepen even more for large frequencies and wave numbers, so it might be that the results reported in reality are weighted by a contribution from a production subrange. 
Table 1. Spectral results from laboratory experiments. The data from Tore Supra identify a production and a coupling subrange as indicated (Hennequin et al., 2004). The results are supported by different discharges giving nearly similar power spectra. A Q-machine experiment (Mikkelsen and Pécseli, 1978; Pécseli et al., 1983) also identifies a production and a coupling subrange.

\begin{tabular}{llccc}
\hline Source & Device & $\begin{array}{c}\text { Spectral indices, } \\
\text { density }\end{array}$ & $\begin{array}{c}\text { Spectral indices, } \\
\text { potential }\end{array}$ & $\begin{array}{c}\text { Spectral indices, } \\
\text { electric field }\end{array}$ \\
\hline Bol (1964) & Etude stellarator & $4.8 \pm 0.2$ & - & - \\
D'Angelo and Enriques (1966) & Q machine & - & $5 \pm 0.2$ & - \\
Robinson and Rusbridge (1971) & ZETA discharge & - & - & - \\
Smith and Powers (1973) & RF discharge & $5 \pm 0.2$ & - & - \\
Mikkelsen and Pécseli (1978); Pécseli et al. (1983) & Q machine & $1.6 / 5 \pm 0.2$ & $2.2 / 5 \pm 0.2$ & - \\
Beall et al. (1982) & RF discharge & - & $2.7 \pm 0.3$ & - \\
Levinson et al. (1984) & Tokamak & $2.7 \pm 0.3$ & $2.7 \pm 0.3$ & - \\
Latten et al. (1995) & KIWI device & $3.6 \pm 0.1$ & - & - \\
Tynan et al. (2006) & CSDX device & $5.4 \pm 0.2$ & - & - \\
Hennequin et al. (2004) & Tore Supra & $3.5 / 6.5$ & - & - \\
Yamada et al. (2010) & Mirror device & 7 & - & - \\
\hline
\end{tabular}

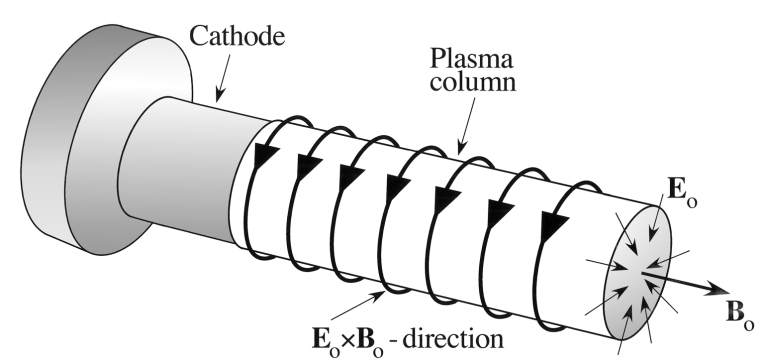

Figure 4. Schematic illustration of a Q-machine set-up with a nearly "solid body" $E_{0} \times \boldsymbol{B}$-rotating plasma column induced by a radial DC electric field $E_{0}$ (Mikkelsen and Pécseli, 1978). The hot filament ("cathode") was made by a double tantalum spiral, heated by a DC current. Because of its spiral structure, the filament imposed an almost parabolic potential variation across the plasma column, giving rise to an imposed electric field, $E_{0}$, increasing nearly linearly with radius (Pécseli et al., 1983).

For scales comparable to the density gradient scale length, the assumption of local isotropy can be difficult to justify. For short wavelengths it was found by microwave scattering (Okabayashi and Arunasalam, 1977) that local isotropy in the plane perpendicular to $\boldsymbol{B}$ could be argued. Physically, this implies that short wavelength drift waves propagate on the density gradients of longer wavelengths, which in turn propagate on gradients originating from even larger spatial scales, etc. For a long-wavelength "production subrange", it seems more natural to assume that the waves propagate in one preferred direction, given by the electron diamagnetic drift velocity $\boldsymbol{U}_{\mathrm{De}}$. The two-dimensional nature of drift wave fluctuations in a plane perpendicular to the magnetic field was confirmed, for instance, by microwave scattering also in the ohmic discharges in the Tore Supra tokamak (Truc et al., 1992).

For neutral turbulence it has been argued by Tennekes (1975) that large eddies sweep small eddies past a fixed ob-

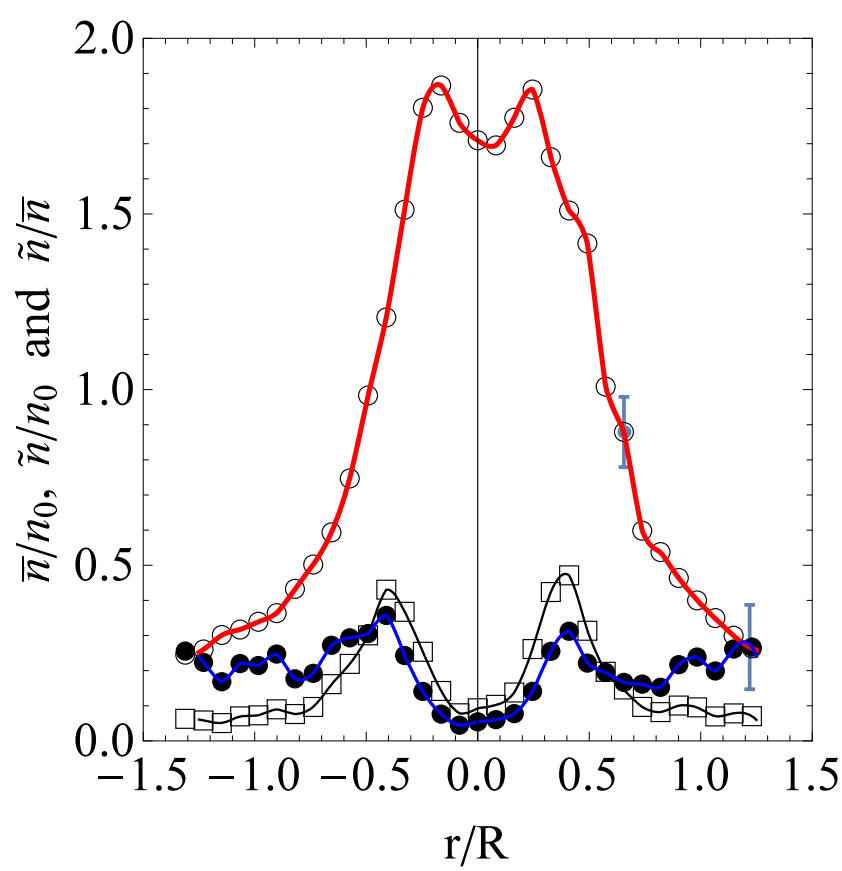

Figure 5. Summary of basic Q-machine caesium plasma conditions (Pécseli et al., 1983); see also Fig. 4. Temperatures were $T_{\mathrm{e}} \approx 2 T_{i} \approx 0.2 \mathrm{eV}$ and magnetic fields $\boldsymbol{B} \in\{0.1-0.25\} \mathrm{T}$. The symbols "O" give the average density $\bar{n} / n_{0}$; " $\square$ " gives the root-meansquare (RMS) fluctuation level $\sqrt{\left\langle\left(\tilde{n} / n_{0}\right)^{2}\right\rangle}$; both are normalized by $n_{0}=10^{10} \mathrm{~cm}^{-3}$. The relative RMS-fluctuation level $\sqrt{\left\langle(\tilde{n} / \bar{n}(r))^{2}\right\rangle}$ by shown with " $\bullet$ ". The normalizing length scale is $R \approx 4 \mathrm{~cm}$. The turbulent fluctuations make the plasma column expand outside the radius of the plasma-producing hot filament, so we find plasma also for radial positions larger than $R$.

server at a velocity that is sufficiently large to make the Taylor hypothesis applicable the small scales, giving a $\omega^{-5 / 3}$ spectrum for large frequencies; see also the discussion of 


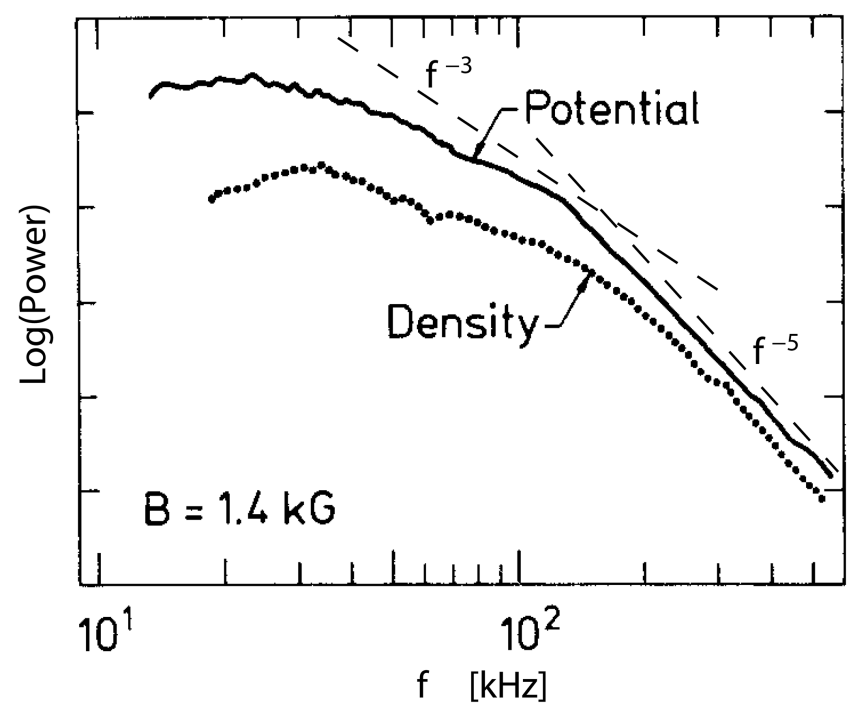

Figure 6. Experimentally obtained power spectra for fluctuations in density and potential (Pécseli et al., 1983). The spectra are obtained at a position $r=0.6 R$ in Fig. 5. Note the similarity between the density and potential spectra, in particular for large frequencies. Dashed lines give $f^{-3}$ and $f^{-5}$ for reference.

Eq. (3). Isotropy on the small scales of the turbulence is essential for this argument since the large-scale motion can have any direction. It is not evident that a similar argument applies to drift wave turbulence. In a weak-turbulence limit, the assumption is definitely questionable, but its use might be justified nonetheless if a linear isotropic dispersion relation with frequency and wave number being proportional (as for ion acoustic sound waves) can be argued.

The variation in the spectral index in the coupling subrange in the terminology of Tchen (1975) and Tchen et al. (1980) is shown in Fig. 7. In the central part of the plasma column we find an index $\alpha \sim 3-4$ but with modest fluctuation levels; see Fig. 5. At positions where the plasma density gradient is substantial and the fluctuation level is enhanced, we have $\alpha \sim 5$ for a wide range of radial positions consistent with a model assuming local homogeneity of the smallscale fluctuations in a plane perpendicular to $\boldsymbol{B}$. The most easily varied parameter was the magnetic field, here in the range of $0.1-0.25 \mathrm{~T}$. Results for two $\boldsymbol{B}$ values are shown in Fig. 7. A change in plasma density would change the resistivity and thereby the linear growth rate of the fluctuations. Plasma density enters the electron-ion collisional resistivity only through the Spitzer logarithm, so this variation is weak (Chen, 1984). Variations in plasma temperature will have a stronger effect.

In laboratory experiments we have the additional constraint that the average collisional mean free path $\ell_{c}$ should be much smaller than the length of the device. Since $\ell_{c} \sim$ $T^{2} n^{-1}$ for electron-ion collisions in terms of temperatures $T$ and plasma densities $n$, most laboratory studies of resis-

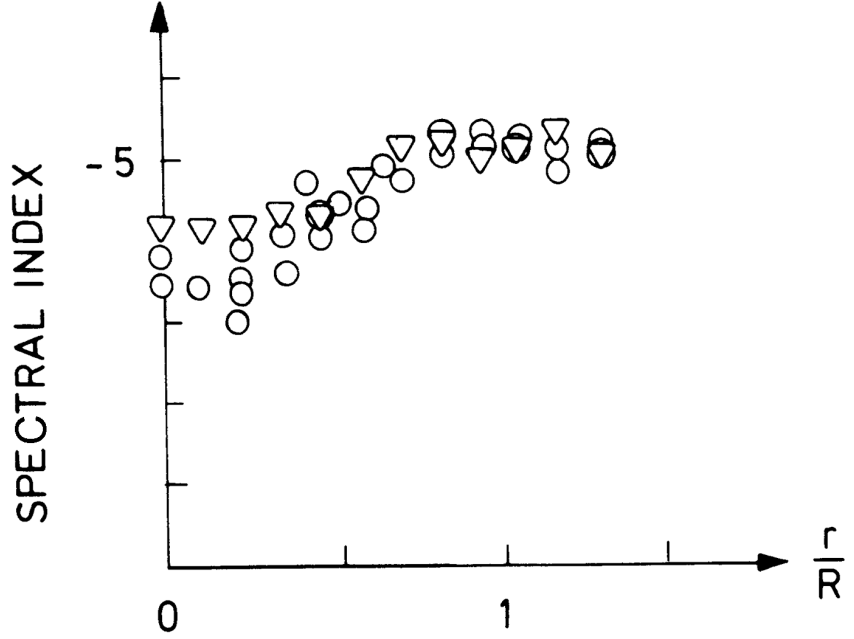

Figure 7. Variation in the spectral index $\alpha$ in the "coupling subrange" for electrostatic potential based on the high-frequency parts of spectra as that shown in Fig. 6 obtained at different radial probe positions. Results from two different magnetic field values are indicated by "O" and " $\nabla$ ". Radial positions are normalized by the outer radius $R=4 \mathrm{~cm}$ of the terminating hot spiral that produces the plasma; see Fig. 4. At the same time it imposes the radial electric field that gives the near solid-body plasma rotation (Pécseli et al., 1983). The scatter in data represents the uncertainty.

tive electrostatic drift waves have been carried out at relatively large plasma densities. Enhanced growth rates of drift wave instabilities can be found with field-aligned currents (Hatakeyama et al., 1980, 2011; Garcia and Pécseli, 2013): in these cases the restriction on mean free paths is of minor consequence.

In comparison to the spectral index $\sim 5$ mentioned above, some related experiments for resistive drift wave turbulence (Yamada et al., 2010) found somewhat steeper frequency spectra with an index as high as 7 for the fluctuations in plasma density as measured by the ion saturation current to a probe. Density fluctuation spectra from ohmic discharges in the Tore Supra tokamak using $\mathrm{CO}_{2}$ laser scattering for diagnostics (Gürcan et al., 2009) gave similar results, in agreement with results based on analytical "shell" models of turbulence. In these studies the spectrum is seemingly defined as in Eq. (14) rather than in the reduced form used in Eq. (7), and there need not be significant disagreement with the drift wave index 5 .

A finite length of an experimental device imposes certain restrictions on the wavelengths parallel to $\boldsymbol{B}$ so that the range of variability in $\sigma_{\|}$is limited (Chen, 1965b, c, 1979); see also Fig. 2, where the parts with large abscissa values are relevant for most laboratory conditions. As a consequence, the growth rate of resistive drift waves is seldom large in laboratories. For small-amplitude linear waves in this limit, we have $e \widetilde{\phi} / T_{\mathrm{e}} \approx \widetilde{n} / n_{0}$ and a small phase difference between the fluctuating density and potential; see Fig, $2 \mathrm{~b}$ and c. Strong 
drift wave turbulence levels in laboratory plasmas are usually found when some additional free energy is available due to rotation (Mikkelsen and Pécseli, 1978). Due to the difference in electron and ion centrifugal forces at high $\boldsymbol{E}_{0} \times \boldsymbol{B} / B^{2}$ angular velocities (see Fig. 4), the electrons and ions do not have the same azimuthal velocity (Odajima, 1978; Mikkelsen and Pécseli, 1978), and this rotation difference gives rise to an azimuthal current that increases the available free energy for the instability. Moreover, a velocity shear via KelvinHelmholtz instabilities enhances the drift wave growth rates. Only sheared velocities in the direction transverse to the magnetic field lines are relevant in the present context (Kent et al., 1969; Jassby, 1972; Nielsen et al., 1992). Sheared plasma flows along magnetic field lines (D'Angelo and von Goeler, 1966) will in general give strongly anisotropic threedimensional turbulence.

\subsection{The influence of collisions with neutrals}

Results from laboratory studies of plasmas with varying neutral background densities have also been carried out (Mikkelsen and Pécseli, 1980). In this case a parabolic potential variation was imposed externally on the hot-plate position of a Q machine (Motley, 1975) as in Sect. 3.1. The resulting stationary electric field gave rise to a zero-order solidbody rotation of the plasma column (Pécseli et al., 1983). A similar experiment was carried out in discharge plasma (John and Saxena, 1975; Prasad et al., 1994) with very similar results. Results for experimentally obtained variations in power spectra for varying neutral density are shown in Fig. 8. The variations in ion-neutral collision frequency correspond qualitatively to what is found by moving from the top of the ionospheric $\mathrm{F}$ region into the lower parts of the $\mathrm{E}$ region.

Two features are conspicuous in Fig. 8: a flattening of the spectra for both density and potential fluctuations and an increasing difference between the spectral index for density and potential as the collision frequency between charged particles and neutrals is increased. The density fluctuation level increases as the neutral density increases. At high neutral collisionalities, as also found in the ionospheric $\mathrm{E}$ region, the ions are no longer magnetized and it can be expected that the power spectra differ significantly from those observed with magnetized ions.

For low neutral densities we find that the spectral indices for density and potential are nearly equal, consistent with a model where $\widetilde{n} \sim \widetilde{\phi}$, resulting from an approximate local Boltzmann distribution of isothermal electrons. As the neutral density (and thereby the collision frequency of charged particles and neutrals) is increased, we find the density spectrum to be "flatter" than the potential spectrum, with a difference in index close to 2 . This limit is consistent with models where $\widetilde{n} \sim \widetilde{E}$, a result found also by analysing data from E region rockets (Pfaff et al., 1987b). The electron-neutral collisions inhibit the free flow of electrons along magnetic field lines, and a local isothermal electron Boltzmann relation can
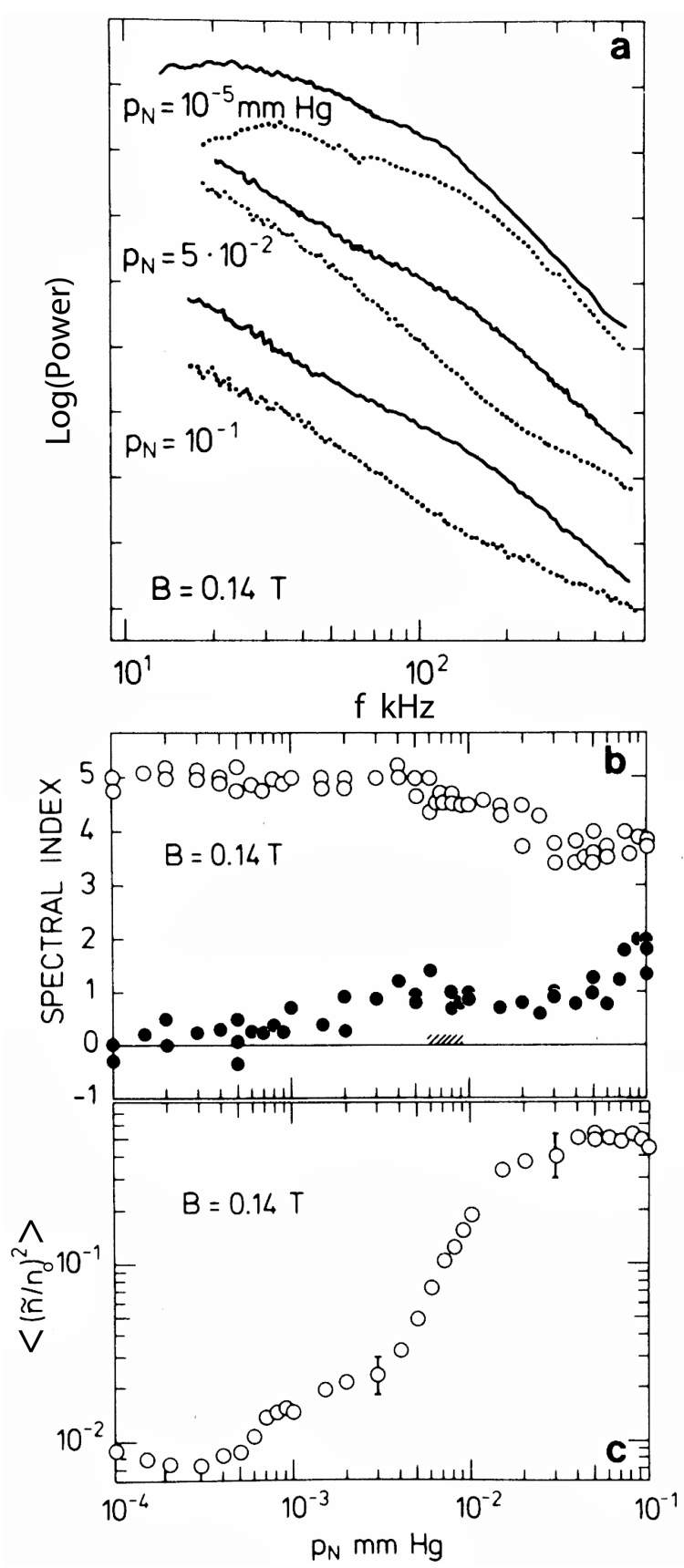

Figure 8. Power spectra for the electrostatic potential and plasma density at different neutral background pressures are shown in (a), where full lines refer to potential and dotted lines to density fluctuations. The spectral index for the potential is shown in (b) with "O" for varying neutral pressures, while "•" gives the difference between the spectral indices for density and potential fluctuations. A shading on the horizontal axis in (b) indicates where the ion cyclotron frequency equals the ion-neutral collision frequency. In (c) we have the variation in mean square relative density fluctuation $\left\langle\left(\widetilde{n} / n_{0}\right)^{2}\right\rangle$ obtained at a fixed radial position for varying neutral pressures (Mikkelsen and Pécseli, 1978). The data were obtained in the same experimental set-up as the one used for Fig. 6. 
no longer be established. The spectral indices of density and electric field fluctuations become similar in this limit.

The RMS-density fluctuation level $\sqrt{\left\langle\left(\tilde{n} / n_{0}\right)^{2}\right\rangle}$ increases significantly with increasing neutral density, from approximately $10 \%$ to approximately $70 \%$ as observed at a fixed radial position; see Fig. 8c. The power spectra reported in the rocket studies mentioned before (Pfaff et al., 1987b) have a large uncertainty, but they are not inconsistent with laboratory results at enhanced neutral pressures (Mikkelsen and Pécseli, 1980; Prasad et al., 1994).

When the linear Farley-Buneman instability (Buneman, 1963; Farley, 1963) is strong (see discussion in Sect. 4.1), it might excite short wavelengths directly, without any intervening nonlinear spectral cascade found in strong-turbulence models. This process gives rise to the flattening of both density and potential spectra as the relative electron-ion drift exceeds the sound speed and increases.

By a narrow-band filtering of the data at enhanced pressures, a dispersion relation between frequency and propagation velocity could be obtained (Mikkelsen and Pécseli, 1980), which is tantamount to a dispersion relation. In the sense discussed in Sect. 2.2, the fluctuations may not represent a strongly turbulent state in this case, in spite of the strongly enhanced fluctuation level.

The discrepancy between the two data sets for RF discharges noted in Table 1 can be due to different radial electric fields for the two cases. These discharges are often operated at relatively high neutral pressures, and it is plausible that the results of one case (Beall et al., 1982) in reality correspond to the high neutral pressures depicted in Fig. 8b.

\section{Drift waves in the ionosphere}

Wave studies in the ionosphere have the attractive feature of allowing boundary conditions to be ignored in many cases. The analysis of collisional or resistive drift waves applies to the Earth's ionosphere. Collisions between charged particles and neutrals dominate collisions between electrons and ions at low altitudes, while results for resistive drift waves will apply at higher altitudes. In the equatorial regions, the geometry will be the standard, with density gradients perpendicular to the magnetic field lines. In polar regions there will be a density gradient component along magnetic field lines as well, but simplified models have been suggested by Garcia and Pécseli (2013) which can account for this feature, too. This particular problem is interesting because it includes an example of a "fake instability", where a seeming wave amplification is due to conservation of wave energy flux in a medium with decreasing density (Dysthe et al., 1975, Pécseli, 2012).

\subsection{Linear dispersion relations for ionospheric $\mathrm{E}$ region parameters}

The ionospheric plasma in the $\mathrm{E}$ and lower parts of the $\mathrm{F}$ region has a significant neutral component. For a wide altitude range up to $110-120 \mathrm{~km}$, the collisions between neutrals and charged particles will completely dominate electron-ion collisions so that the ions are in effect unmagnetized. When we have $\omega_{\text {ce }} \gg v_{\text {en }}$ while $\Omega_{\text {ci }} \leq v_{\text {in }}$, in terms of electron and ion cyclotron frequencies $\omega_{\mathrm{ce}}$ and $\Omega_{\mathrm{ci}}$ with electron-ionneutral collision frequencies $\nu_{\mathrm{en}}$ and $\nu_{\text {in }}$, respectively, we have significantly different Hall mobilities of ions and electrons. These conditions have no direct counterpart in the classical analysis of resistive drift waves (Chen, 1965d, e, 1984). One consequence of the difference in mobilities is the possibility of driving a significant Hall current by an externally imposed electric field $E_{0} \perp \boldsymbol{B}$. The steady-state electric field $E_{0}$ can be imposed by the interaction between the solar magnetic field and the Earth's magnetosphere (Kelley, 1989).

For the present problem we find a linear dispersion relation for low-frequency electrostatic waves in the form (Fejer et al., 1975; Kelley, 1989) valid in the limit where $\omega_{\text {re }} \leq v_{\text {in }}$ and $\omega_{\text {im }} \ll \omega_{\text {re }}$ with the real part of the frequency being

$\omega_{\mathrm{re}} \approx \frac{\boldsymbol{k} \cdot\left(\boldsymbol{U}_{e 0}+\Psi \boldsymbol{U}_{i 0}\right)}{1+\Psi}$

To the same approximation, the imaginary part of $\omega$ is obtained as

$$
\begin{aligned}
\omega_{\mathrm{im}} \approx & \frac{1}{1+\Psi}\left\{\frac{\Psi}{v_{\mathrm{in}}}\left(\left(\omega_{\mathrm{re}}-\boldsymbol{k} \cdot \boldsymbol{U}_{i 0}\right)^{2}-k^{2} C_{\mathrm{s}}^{2}\right)\right. \\
& +\frac{1}{k^{2} L}\left[\left(\omega_{\mathrm{re}}-\boldsymbol{k} \cdot \boldsymbol{U}_{i 0}\right)\left(\frac{v_{\mathrm{in}}}{\Omega_{\mathrm{ci}}} k_{y}+\Psi_{0} k_{z}\right)\right. \\
& \left.\left.-\left(\omega_{\mathrm{re}}-\boldsymbol{k} \cdot \boldsymbol{U}_{e 0}\right)\left(\frac{v_{\mathrm{in}} \Omega_{\mathrm{ci}}}{v_{\mathrm{in}}^{2}+\left(\omega_{\mathrm{re}}-\boldsymbol{k} \cdot \boldsymbol{U}_{i 0}\right)^{2}} k_{y}-k_{z}\right)\right]\right\} \\
& -2 \alpha n_{0},
\end{aligned}
$$

with

$$
\Psi \equiv \frac{v_{\mathrm{in}} v_{\mathrm{en}}}{\Omega_{\mathrm{ci}} \omega_{\mathrm{ce}}}\left(\frac{k_{\perp}^{2}}{k^{2}}+\frac{\omega_{\mathrm{ce}}^{2}}{v_{\mathrm{en}}^{2}} \frac{k_{z}^{2}}{k^{2}}\right) \equiv \Psi_{0}\left(\frac{k_{\perp}^{2}}{k^{2}}+\frac{\omega_{\mathrm{ce}}^{2}}{v_{\mathrm{en}}^{2}} \frac{k_{z}^{2}}{k^{2}}\right)
$$

and ion/electron steady-state drift velocities being $\boldsymbol{U}_{i 0}$ and $\boldsymbol{U}_{e 0}$, respectively, while $|L| \equiv\left|n_{0} / \nabla n_{0}\right|$ is the zero-order length scale of the background unperturbed density gradient. The sign of $L$ is measured with respect to $E_{0}$. The gradient term contributes to an instability when $\boldsymbol{E}_{0} \cdot \nabla n_{0}>0$. The first term in Eq. (17) accounts for the Farley-Buneman or Hall current instability (Buneman, 1963; Farley, 1963), which can be effective even in the absence of a zero-order density gradient. This instability requires the relative Hall drift of the electrons with respect to the ions to exceed the ion acoustic sound speed. The current-driven instability excites predominantly short wavelengths in contrast to the gradient instability. If, on the other hand, the relative ion-electron Hall drift 
is less that the sound speed, this first term in Eq. (17) acts as a damping, predominantly for short wavelengths. The requirement $\omega_{\text {im }}>0$ defines a directional cone (not necessarily with elliptical cross section) in wave number space where enhanced fluctuations are supported. Waves propagating outside this cone are generated by nonlinear interactions.

A model consistent with the observations is one where we have a $k^{-5}$ drift wave turbulent spectrum at low neutral pressures with negligible Hall current. As the collision frequency between plasma particles and neutrals increases (for increasing neutral pressure), the Farley-Buneman instability is excited in the rapidly rotating plasma (most effectively at the edge of the plasma column) and generates predominantly large wave numbers to flatten the drift wave spectra for both density and potential fluctuations. For large neutral pressures the neutral drag on the ions is effective, the ion-electron velocity difference exceeds the ion sound speed and the Hall current instability dominates entirely in the large frequency limit; see Fig. 8. The enhanced density fluctuation level for increasing Hall current, observed in Fig. 8, indicates that the current instability is more effective than the drift instability, at least in that particular experiment. For large Hall currents the power spectrum is thus dominated by the two-stream instability contribution and the fluctuations will also be excited to significant amplitudes in the absence of any density gradient. In this limit the nearly two-dimensional features of the turbulence may also vanish, although the electrojet instabilities are usually found to have a high aspect angle sensitivity (Foster et al., 1992), at least for short wavelengths. Laboratory experiments (D'Angelo et al., 1974; John and Saxena, 1975; Mikkelsen and Pécseli, 1980) will inevitably have density gradients perpendicular to the confining magnetic field. The polarity of this field was fixed by the experimental conditions, and it was not possible to distinguish the contributions from the Farley-Buneman and gradient instabilities in the experiments. This could have been possible if a change in sign of $E_{0} \cdot \nabla n_{0}$ had been feasible in the experiment.

\subsection{Space observations}

Turbulent spectra have been observed in situ in the Earth's ionosphere by use of instrumented rockets and satellites. Following the set-up used elsewhere (Kintner and Seyler, 1985), we summarize selected results in Tables 2 and 3, with parts of the information summarized in Figs. 9 and 10. The scatter in spectral indices is large, even within one experiment, and it is recommended that the original papers are consulted to see precisely what result they are being cited for. Note the correction of spectral indices (Prakash et al., 1971) that takes into account a varying filter bandwidth: this reference is predated by others (Prakash et al., 1968, 1969), containing an erroneous value for spectral indices. Values for positive spectral indices have been reported by Prakash et al. (1971), but these apply only to some unspecified narrow spectral subranges and they are not included in the present summary. Some rocket experiments (Pécseli et al., 1989, 1993; Iranpour et al., 1997) report $E$ region turbulent spectra in a colour-coded version, where a spectral index is not readily determined with any significant accuracy. Within the uncertainty, the results do not, however, in contradiction with similar data in Table 2. Observations of turbulent electric field spectra have been obtained by instrumented balloons (Mozer, 1971), but these data will not be discussed further here, although they can be relevant for comparison with $\mathrm{E}$ region turbulence results. Unfortunately, often only an altitude range is given for a satellite experiment, without specifying the precise altitude where the power spectra were obtained.

Since rockets or satellites do not have an absolute ground, potential variations are usually detected by using probe potential differences. For scales or wavelengths much larger than the probe separation, the measurements can be interpreted in terms of an electric field component taken along the direction of the boom connecting the probes. Very often the situation is the opposite: the scales are shorter than the probe separations. This has consequences for the interpretation of turbulent potential spectra detected by probes. The Appendix offers arguments for interpreting high-frequency, short-wavelength parts of experimentally obtained spectra as power spectra for the electric potential.

A vast number of numerical simulations contain partial results for spectral power laws: there are too many to be summarized here, in part also because it is not always evident which ionospheric parameter range (if relevant at all) the analysis refers to. There is an early summary of studies of the type II irregularities (McDonald et al., 1974), giving a density fluctuation spectrum close to $k^{-3.5}$, albeit with a clear tendency for steepening at small wavelengths. It is stated explicitly that this result is obtained by averaging over all directions, so that a $k$ integration directly gives $\left\langle\widetilde{n}^{2}\right\rangle$. Some studies (Gondarenko and Guzdar, 2004) compare the results of numerical simulations with experimental observations (Basu et al., 1990) and emphasize a subrange that would correspond to the production subrange in the terminology of Tchen et al. (1980). This subrange is, however, in the simulations of Gondarenko and Guzdar (2004) followed by a "steeper" subrange that is not discussed in detail, but it might be noted that it has the characteristics of a coupling subrange.

An overview and synthesis of plasma irregularities and turbulence was given by Hysell (2000), with emphasis on the F region.

Some reports seem to distinguish drift and transverse velocity shear-driven turbulence (Kintner and Seyler, 1985; Basu et al., 1988). Indeed in what could be called the production range, we might expect differences. For an inertial (or coupling) subrange, the precise nature of the energy input should be of little consequence in the strong-turbulence limit, since the spectral shape would be expected to be controlled by the cascade, which will be the same for small-scale electrostatic drift and $\boldsymbol{B}$-transverse velocity shear instabilities. The theoretical analysis of Tchen et al. (1980) antici- 
Table 2. Observations made by instrumented rockets. If two values for a spectral index are listed, then the lowest frequency range is listed first. Note that the uncertainty on the spectral estimates is not always given in the literature. The data are sorted according to average altitudes, with the lowest altitudes first.

\begin{tabular}{lrccc}
\hline Source & Region/altitude & $\begin{array}{c}\text { Spectral indices, } \\
\text { density }\end{array}$ & $\begin{array}{c}\text { Spectral indices, } \\
\text { potential }\end{array}$ & $\begin{array}{c}\text { Spectral indices, } \\
\text { electric field }\end{array}$ \\
\hline Pfaff et al. (1987a) & $92-105 \mathrm{~km}$ & - & - & $1.2 / 4.5$ \\
Krane et al. (2000) & $100-110 \mathrm{~km}$ & $1 \pm 0.25$ & $3 \pm 0.3$ & - \\
Prakash et al. (1971) & $150 \mathrm{~km}$ & - & - & $2.5 \pm 0.5$ \\
LaBelle et al. (1986) & $300-500 \mathrm{~km}$ & $2.5 / 4.5$ & - & - \\
Kelley et al. (1982a) & $390-560 \mathrm{~km}$ & $2.2 \pm 0.2 / 5.5 \pm 0.5$ & - & - \\
Earle et al. (1989) & $450-500 \mathrm{~km}$ & $2.2 \pm 0.5$ & - & $2.9 \pm 0.5$ \\
Kelley (1982); Kelley et al. (1982b) & $500 \mathrm{~km}$ & $4.5 \pm 0.4$ & - & $3.1 \pm 0.3$ \\
\hline
\end{tabular}

pated this by having two different "production mechanisms" for the respective subranges of velocity and potential fluctuations. The hypothesis finds support in numerical simulations of nonlinear evolution of the Kelvin-Helmholtz instability in the high-latitude ionosphere (Keskinen et al., 1988), where the high-frequency short-wavelength part of the spectrum was found to have characteristics very similar to those of electrostatic drift waves. Similar studies for the ion temperature gradient (ITG) instability have not been carried out with the same aim and to the same accuracy, but such a study seems worthwhile in the light of the results mentioned above.

The results of Basu et al. (1990) for the electric field in Table 3 are obtained for different orbits, with a seemingly large variability. It may be that the variation is due to different subranges being sampled. This work includes an interesting comparison of spectra for different wave number component directions perpendicular to the magnetic field, giving some indications of local isotropy in that plane. These results were compared to numerical results from turbulence simulations (Keskinen and Huba, 1990), and for some of the data, the agreement was within the uncertainties.

The lists in Tables 2 and 3 are by no means exhaustive, but already on this limited basis we can state that the results are not unconditionally promising regarding a universal turbulent spectrum. There is a scatter in the spectral indices reported, although this is seen mostly for the satellite data; see Fig. 10. Separating data from rocket and satellite experiments (as in Figs. 9 and 10), it becomes easier to obtain a coherent presentation. The rocket data will thus support models for a coupling subrange where a power law index near 5 for density and an index of 3 for electric field fluctuations is found for high altitudes and thereby low neutral collisionalities, $\Omega_{\mathrm{ci}}>v_{\mathrm{in}}$. The observed spectra (Earle et al., 1989) giving a power law index of 2.2 for the density may be a misinterpretation, belonging in reality to a large amplitude production subrange where an index of 3 is predicted (Tchen et al., 1980). The remaining disagreement with results expected for electrostatic drift wave turbulence are then be the observations from the E region (Pfaff et al., 1987a; Krane et al., 2000), where both potential and density spec-

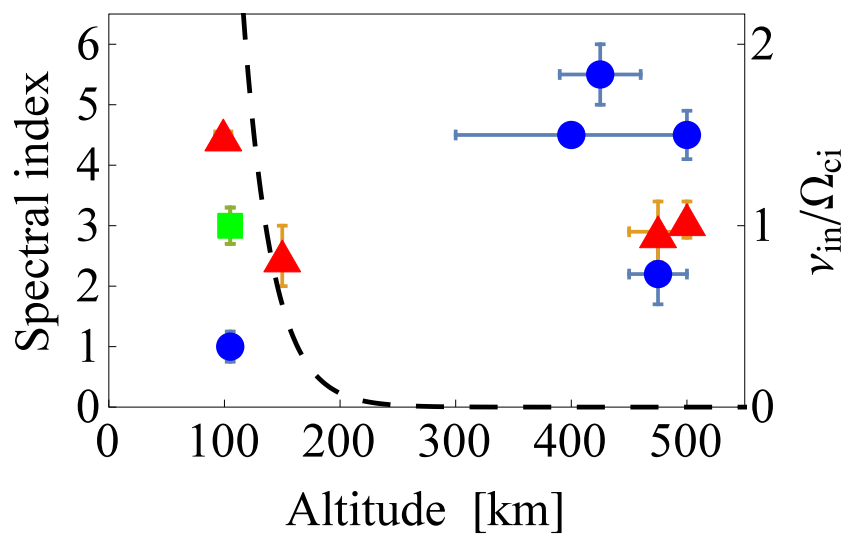

Figure 9. Colour-coded representation of parts of the information in Table 2. The power spectral index variation with altitude for density is shown as blue circles, electric field as red triangles and one measurement for potential (Krane et al., 2000) as a green rectangle; see also the discussion in the Appendix. Only spectral indices for the (assumed) coupling subranges are shown. The dashed line (with scale to the right of the figure) shows the normalized altitude variation in the ion-neutral collision frequency $v_{\mathrm{in}}(z) / \Omega_{\mathrm{ci}}$

tra are completely off. This limit can, however, be accounted for with reference to laboratory results of Mikkelsen and Pécseli (1980), where the neutral collisions and the effects of the Farley-Buneman (FB) instability modify the spectra. In Fig. 9, a dashed curve shows the normalized altitude variation in the ion-neutral collision frequency $\nu_{\text {in }}(z) / \Omega_{\mathrm{ci}}$. This curve will change with plasma conditions and vary in particular for day and night conditions. The corresponding electron ratio $v_{\mathrm{en}}(z) / \omega_{\mathrm{ce}}$ in negligibly small in the altitude range shown. The curve is based on an average ion mass of 31 AMU and a vertical scale length $C_{\mathrm{s}}^{2} / g$ of $25 \mathrm{~km}$ for a sound speed of $C_{\mathrm{s}}=500 \mathrm{~m} \mathrm{~s}^{-1}$. For the altitude range shown in Fig. 9, we can take the magnetic field to be constant.

The altitude range with parameters $\Omega_{\mathrm{ci}} \ll \nu_{\text {in }}$ should be considered for two separate cases: one where the Hall electron drift is smaller than $C_{\mathrm{s}}$ and one where it is larger. The laboratory experiment of Mikkelsen and Pécseli (1978) sum- 
Table 3. Observations made by instrumented satellites. The results are obtained by determining the exponent $\alpha$ in frequency spectra $\sim f^{-\alpha}$ as in Table 2. For the data given by Kintner (1976) only the high-intensity cases are cited. The data are sorted according to average altitudes, with the lowest altitudes first. Some studies, e.g. by Kintner (1976), also report magnetic field fluctuations with power law spectra. If these fluctuations represent propagating waves, their presence may indicate relatively large plasma $\beta$ values. The magnetic field fluctuations need not always follow a power law spectrum (Gurnett and Frank, 1977).

\begin{tabular}{lrccc}
\hline Source & Region/altitude & $\begin{array}{c}\text { Spectral indices, } \\
\text { density }\end{array}$ & $\begin{array}{c}\text { Spectral indices, } \\
\text { potential }\end{array}$ & $\begin{array}{c}\text { Spectral indices, } \\
\text { electric field }\end{array}$ \\
\hline Shume and Hysell (2004) & $325-450 \mathrm{~km}$ & $1.6 \pm 0.05$ & - & - \\
Basu et al. (1990) & $400 \mathrm{~km}$ & $1.9 \pm 0.1$ & - & $1.9 \pm 0.1$, \\
& & & $2.3 \pm 0.1$, \\
Rodrigues et al. (2009) & $500 \mathrm{~km}$ & $5.5 \pm 1$ & - & $3.3 \pm 0.1$ \\
McDaniel and Hysell (1997) & $530 \mathrm{~km}$ & $1.65 / 3.52$ & - & $1 \pm 1$ \\
Dyson et al. (1974) & $440-780 \mathrm{~km}$ & $2.0 \pm 0.2$ & - & - \\
Cerisier et al. (1985) & $400-2000 \mathrm{~km}$ & 1.93 & - & - \\
Golovchanskaya et al. (2012) & $700-2500 \mathrm{~km}$ & - & - & $2.2 \pm 0.3$ \\
Gurnett and Frank (1977) & $24000-30000 \mathrm{~km}$ & - & - & $3 \pm 0.3$ \\
Angelopoulos et al. (2001) & $5700-54000 \mathrm{~km}$ & - & - & $1 / 2.5$ \\
Kintner (1976) & $500-125000 \mathrm{~km}$ & - & $2.8 \pm 0.3$ \\
\hline
\end{tabular}

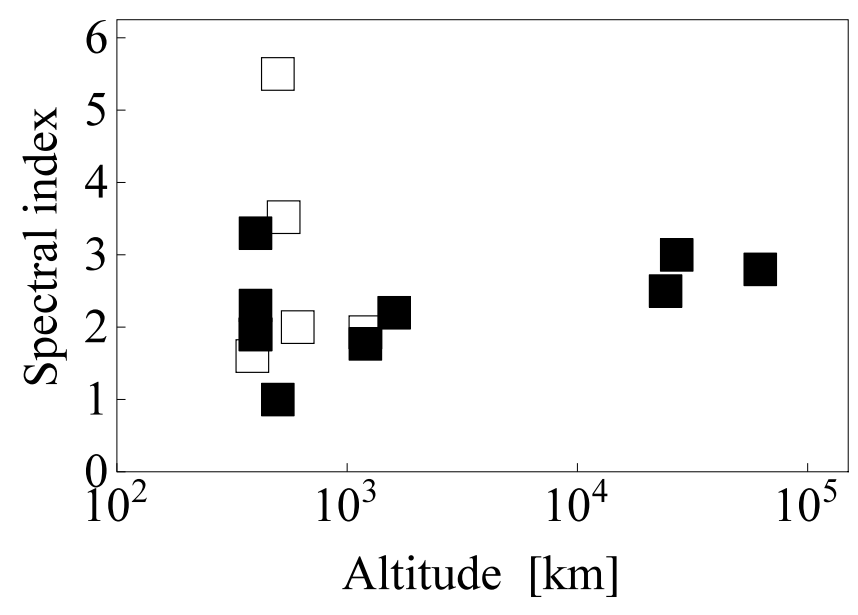

Figure 10. Black- and white-coded representation of parts of the information in Table 3. The power spectral index variation with altitude (on a logarithmic scale) for density is shown as " $\square$ " and for electric field as " $\mathbf{\square}$;. The error bars are large, in particular for the altitudes (see Table 3) and are omitted here. Some data are obtained outside the Earth's magnetopause. There are no reports concerning the plasma $\beta$ values for the altitudes where these spectra are obtained.

marized in Fig. 8 refers to the latter case. The distinction arises from the first term in Eq. (17). For the supersonic Hall drifts, it gives rise to enhanced short-wavelength fluctuations. In the subsonic flow the term damps short wavelengths, and gradient drift spectra can be expected to be steeper that those found for conditions with negligible neutral collisions. For the flat spectra the plasma conditions were consistent with the FB instability being excited (Mikkelsen and Pécseli, 1978), while the steeper spectra (Pfaff et al., 1987a) were as- sociated with the gradient drift instability only. Results from a laboratory experiment (Saxena and John, 1975) have a large uncertainty and estimate a power law on the basis of a few modes only, but the results are not in disagreement with the rocket results. These observations relate only to what is here called the coupling subrange: the corresponding production subrange at long wavelengths (see first entry in Table 2) is only marginally affected here (Pfaff et al., 1987a).

Using dimensional analysis Ott and Farley (1974) attempted to give analytical predictions for the wave number power law spectra for turbulent density fluctuations generated by the gradient instability with high neutral collisionality. Defining a spectrum for fluctuating densities $\left\langle\left(\widetilde{n} / n_{0}\right)^{2}\right\rangle \equiv$ $\int S_{F}(k, \Theta) \mathrm{d} k \mathrm{~d} \Theta$, it can be argued by dimensional reasoning that the spectrum must have a form

$k S_{F}(k, \Theta)=F\left(k L_{n}, \frac{U_{\mathrm{d}}}{k D_{a}}, \Theta, \zeta_{1}, \zeta_{2}\right)$,

where $F$ is a dimensionless function of several dimensionless variables, with $L_{n} \equiv n_{0} /\left|\nabla n_{0}\right|, \Theta$ is the angle between $k$ and the relative electron-ion drift velocity $\boldsymbol{U}_{\mathrm{d}}$, while $D_{\mathrm{a}}$ is the ambipolar diffusion coefficient and $\zeta_{1}, \zeta_{2}$ are reduced dimensionless mobilities accounting for the collisional transport in the region with enhanced neutral collisions. The model thus contains several parameters that have to be fitted by experiments. It is found experimentally that the scattered radar power scales approximately with the square of $U_{\mathrm{d}}$. If the other parameters remain constant for varying $U_{\mathrm{d}}$, this observation indicates that $S_{F}(k, \Theta) \sim k^{-3}$ in an inertial subrange, but this does not agree well with the rocket observations (Pfaff et al., 1987a); see Table 2 and Fig. 9. The analysis assumed fluctuations in density and electric field to be proportional. If we make the plausible assumption that 
the power scales inversely proportional to $L_{n}^{2}$, the arguments will imply the existence of a production subrange where $S_{F}(k, \Theta) \sim k^{-3}$. This result corresponds to the production subrange found in other studies (Tchen et al., 1980).

If we also include data from satellites (see Fig, 10), we find a significant scatter in data points, and it seems unlikely that one universal power law can be determined for fluctuations in density, potential or electric fields. The reason is not obvious. It might not be permissible to compare data from such diverse regions of space, but on the other hand, such a comparison should be the whole point of universal properties. The large altitude ranges covered by satellites makes the range of variation in the length scales $L$ for density larger, bearing in mind, however, that the scale lengths should be measured in units of the local effective ion Larmor radius. The neutral density (and thereby the collision frequency between neutrals and plasma particles) decreases rapidly with altitude; see Fig, 9. The electron-ion collision frequency scales as $\sim n T^{-3 / 2}$ and decreases also with altitude above the $\mathrm{F}$ maximum because of the decreasing plasma density $n$ and increasing plasma temperature $T$ there. Ignoring the Spitzer logarithm, we have the plasma resistivity due to electron-ion collisions scaling with temperature as $\sim T^{-3 / 2}$. In general we find that the Spitzer logarithm increases with altitude but not by enough to increase the net plasma resistivity. Consequently, the largest local growth rates for resistive drift waves are found at low altitudes.

Can it be that a universal power law may simply not exist? This conclusion seems somewhat pessimistic, and in the opinion of the present author it is in part a question of sorting the data according to an effective Reynolds number as argued in Sect. 2.2. Moreover, it should be noted that observations of a low-frequency turbulent spectrum need not necessarily imply that the source is a drift instability. Universal laws for strong turbulence will be found when the nonlinear effects dominate, and, with the exception of the results of Kintner (1976), the data are not sorted according to wave intensity. In the one case mentioned, the distinction of low- and high-intensity spectra was significant. Data from the Orbiting Geophysical Observatory 6 (OGO6) (Dyson et al., 1974) showed, however, that for the long-wavelength fluctuations $\left(10-10^{3} \mathrm{~m}\right)$ detected there, the spectral index was constant within the uncertainty for all RMS amplitudes measured. Due to the predominantly long wavelengths involved, these data might originate from a production subrange.

Most laboratory results refer to large intensity fluctuations, and there indications of universal power law spectra were found. It could also be that the instrumentations of the rockets and satellites is insufficient to distinguish between differences in the nature of the turbulence. By applying Taylor's hypothesis (or the frozen turbulence hypothesis) to a "onepoint signal" alone, it is not logically possible to distinguish between different dimensions of the turbulence field. It is particularly unfortunate that with a one-point measurements, it is not possible to estimate the density gradient either; at best only one component of it can be found: for studies of gradient-driven drift wave turbulence this information would be essential, in particular for quantifying a spectral "production subrange".

It is possible that some space observations deal with plasma turbulence that is almost two-dimensional and perpendicular to $\boldsymbol{B}$; other cases may have a driving mechanism that includes components parallel to $\boldsymbol{B}$, where an insufficient instrumentation does not allow for the distinction between the separate cases. In one of the examples considered above (Krane et al., 2000), the rocket was equipped with two booms, giving altogether six signals for probe potential differences (Rinnert, 1992); see also the Appendix. The spacetime resolution of this particular experiment allowed a confirmation of the directionality of the wave propagation (Krane et al., 2000).

\section{Conclusions}

The present summary discussed observations of turbulent fluctuations in magnetized plasmas, studied in the laboratory and in space. It was demonstrated that turbulent spectra can be observed and that these spectra can be characterized by well-defined power laws that can span several orders of magnitude, as in Fig. 6, for instance. No laboratory plasma can, however, come close to the vast spatial ranges found for neutral flows in nature (tidal flows between islands, for instance; Grant et al. (1962)). It might be hoped that space observations can provide such extended power spectral results. So far, even high- $\beta$ turbulence as observed in the solar wind has to rely on composite spectra (found by combining data from several spacecraft) for producing results that cover many orders of magnitude in the power spectrum of magnetic field fluctuations (Bruno and Carbone, 2005).

Turbulence in plasmas dominated by collisions between charged particles and neutrals seem to offer a problem that is not readily accounted for by strong-turbulence models for drift waves. Analytical studies (Hamza and St-Maurice, 1993a, b) can account for the parameter dependence of the RMS-fluctuation levels and other properties but fail, so far, to predict power spectra. For the time being, the most detailed information is obtained by experimental observations. Indications have been found for universal properties of weak turbulence results in neutral-collision-dominated plasmas, with results seemingly in agreement with spectra obtained for current-driven turbulence (Kadomtsev, 1965). To some extent the agreement between these early analytical results (Kadomtsev, 1965) and observations (Machalek and Nielsen, 1973) might seem surprising, since the physical conditions are different. The common feature is, however, that in both cases the turbulence is driven by a current due to a bulk electron drift with respect to ions. In the case studied by Machalek and Nielsen (1973), the current is across magnetic field lines. A detailed (future) analysis has to account not 
only for the power spectrum of potential but also for the observed difference between density and potential fluctuations for the case where instabilities are driven by Hall currents in a magnetized plasma.

It is interesting to note that the rocket data in Table 2 at least do not contradict the variation in spectral index illustrated in Fig. 8 if we associate a decreasing neutral collision frequency with increasing ionospheric altitude. This similarity assumes, however, that the results for the $450-500 \mathrm{~km}$ altitude (Earle et al., 1989) are related to the production subrange of the turbulent spectrum. The scattering in spectral indices becomes conspicuous when satellite data are also included in the database, but up to $\sim 500 \mathrm{~km}$ altitude the rocket and satellite results agree qualitatively for a production subrange. While rocket data give a reasonably coherent picture of the observed spectral indices, satellite data need further scrutiny. It has recently been suggested (Garcia and Pécseli, 2013; Garcia et al., 2015) that the ionospheric plasma at high altitudes can be very sensitive to even small lowaltitude plasma variations when a slow bulk electron flow causes field-aligned currents. Spectra observed at high polar altitudes can thus be influenced by distant low-altitude perturbations. The models assume that the magnetic field lines have a significant vertical component. These comments do not apply for the plasma outside the magnetopause and therefore not to the data for the largest altitudes in Fig. 10. Incidentally it is at these large altitudes that the agreement with predicted spectral indices is good. It may be interesting to establish a database where gradient-driven instabilities in polar and equatorial regions can be distinguished. These observations could be supported also by numerical simulations.

Laboratory experiments seem also to be in reasonable agreement with rocket data concerning spectral indices for low neutral pressures. Detailed studies with enhanced neutral pressures have been carried out but seemingly only for conditions where the Farley-Buneman instability is excited. It would be valuable to investigate cases where only the gradient instability is present, in particular in order to verify that the short-wavelength damping effect of the first term in Eq. (17) does indeed give rise to a steepening of the $k^{-5}$ spectra found for resistive drift waves at negligible neutral pressures. Such an experiment (or numerical simulation) should keep $v_{i} / \Omega_{\mathrm{ci}}$ and $v_{e} / \omega_{\mathrm{ce}}$ constant (in terms of collision frequencies between plasma particles and cyclotron frequencies) and vary the Hall current from $E_{0} / B<C_{\mathrm{s}}$ to $E_{0} / B>$ $C_{\mathrm{s}}$. Moreover, it seems that the fully saturated turbulent development of magnetic field-aligned velocity shear instabilities (D'Angelo and von Goeler, 1966; Koepke and Reynolds, 2007; Koepke, 2008) (possibly weakly turbulent) is not studied in any significant detail and is still not well understood. These instabilities are likely to saturate in a power spectrum that is non-uniformly distributed in a three-dimensional wave vector space.

Turbulent signals can also be found at altitudes below the E region (Røyrvik and Smith, 1984). In these regions we have the neutral collisions dominating the plasma dynamics, and it is generally believed (Gurevich et al., 1997) that turbulence in the neutral background controls the fluctuations of the plasma density. It is not known to what extent incompressible turbulence in the neutral gas can couple to the plasma dynamics in the ionospheric $\mathrm{E}$ region. The study of Buneman (1963) assumes such a coupling but for compressible sound waves. In the presence of local plasma density gradients, even incompressible neutral turbulence will give rise to plasma density fluctuations (Tchen, 1973). The problem was addressed by comparing numerical simulations with rocket data obtained in the ionospheric E region (Dyrud et al., 2006). The agreement was good, and it was argued that since the simulations did not include any neutral gas dynamics, it was unlikely that neutral turbulence had any significant role in the plasma fluctuations observed by the rocket.

Except for a few cases (Machalek and Nielsen, 1973), the observed power spectra are mostly obtained by analysing time records. Wave number spectra are the form most often predicted by analysis, so a comparison relies on Taylor's hypothesis in order to "translate" frequency spectra to wave number power spectra. In a laboratory, it is possible to relate observations to locally homogeneous and time stationary plasma conditions. In nature, a spacecraft will often traverse spatially varying plasma parameters in a relatively short time, so it is possible that a spectral analysis mixes separate spatial regions with different characteristics. The results should be interpreted with this in mind. The local correlation function $R_{\Psi}(\boldsymbol{R}, \boldsymbol{r}, \tau)$ discussed in Sect. 1 and its spatial variation can be analysed both experimentally and by numerical methods to test these features.

Spectra are in general presented with the implied assumption that the turbulence can be considered to be locally homogeneous to a good approximation, possibly also isotropic in two or three spatial dimensions. In this context, it is interesting that non-ideal laboratory studies with widely different plasma conditions give results that show general agreement, indicating that the homogeneity requirement is relatively mild. See for instance Fig. 7, where the spectral index is constant over a significant radial region of the plasma column.

An interesting possibility would be if a spectral index could be used as a means of identifying the instability causing the observed turbulence, but so far this remains speculative. It is interesting to note that turbulence in the high- $\beta$ plasma of the solar wind (Tu and Marsch, 1995; Bruno and Carbone, 2005) has characteristics completely different from those associated with electrostatic drift waves.

For ionospheric and magnetospheric studies in particular, the question of waves excited by moving rockets and satellites also needs to be addressed. Such perturbations can influence the observed wave spectra (Guio and Pécseli, 2005): the moving rocket or satellite can generate the turbulence it detects. 
Low-frequency electrostatic waves in space plasmas, pure drift waves or their modified versions due to the Hall current instability, are important for turbulent transport. For the resistive drift waves, classical models based on transport due to turbulent $\widetilde{\boldsymbol{E}} \times \boldsymbol{B} / B^{2}$ velocities offer a useful starting point (Taylor and McNamara, 1971; Misguich et al., 1987). In this limit both the electron and the ion components move with the same velocity to the lowest order where ion polarization drifts are ignored. The spectral index has important consequences, for instance, for modelling relative turbulent diffusion (Misguich et al., 1987; Mikkelsen et al., 1987), so a coherent analytical model can be useful in this respect. It was found that a $k^{-3}$ power law for the electric field power spectrum had a special role for the relative diffusion of two particles (Misguich et al., 1987). For the limit where collisions with a neutral component dominate (as appropriate for the lower parts of the Earth's ionosphere), the neutral drag on the ions separates the motions of the electron and ion components, requiring a more detailed analysis (Hamza and StMaurice, 1995). Turbulent transport in the ionosphere can be important as it controls the gradients that can build up due to other sources, such as particle precipitation. Enhanced density fluctuations in the ionosphere can also contribute to a scattering of electromagnetic waves or radiation by a wavevector-matching condition (Bekefi, 1966). These waves can be radio waves, GPS signals or naturally occurring radiation. It has thus been argued by D'Angelo (1978) that "cosmic noise absorption events" are in fact not caused by absorption but rather by the backscattering of radiation due to enhanced density fluctuations caused by the combination of drift and Farley-Buneman instabilities (Alport et al., 1981). The twodimensional nature of the turbulence imposes restrictions on the scattering geometry.
As mentioned in the Introduction, higher-order spectra, such as bispectra, can also be of value for interpreting turbulence data. In laboratory studies interesting and important results have been found using these methods (Kim and Powers, 1979; Yamada et al., 2008). Bispectra, in particular, can give indications of phase couplings among selected modes in the turbulence. According to the arguments given in the present summary, evidence for coherent phase relations among a restricted number of modes (evidenced by peaks in the bispectrum) are at variance with strong-turbulence models, where a large number of modes interact at the same time. Bispectral studies have also been attempted for data obtained by instrumented rockets in the ionospheric E region (Pécseli et al., 1993; Larsen et al., 2002; Dyrud et al., 2006). Here, it was found that bispectral couplings were very intermittent or sporadic. The limited data sets made it difficult to obtain quantitative results, but it was interesting that when the same procedures were applied to data from numerical simulations corresponding to similar physical conditions, the results were similar to those found in the rocket data. This bispectral intermittency can be an indicator of a transition from weak to strong turbulence.

Zonal flows (Nagashima et al., 2008; Tynan et al., 2009; Jiquan and Kishimoto, 2011) have not been addressed in the present review. Their observation in space plasmas and the comparison with laboratory results and numerical simulations could be of great interest. 


\section{Appendix A}

Instruments on rockets and satellites usually detect fluctuations in plasma density, for instance by saturation currents to Langmuir probes. These data can be considered as being obtained by "point measurements". Since no absolute ground is available, direct measurements of fluctuating potentials are not generally feasible and only potential differences between two probes can be obtained (Rose et al., 1992), as illustrated in Fig. A1. When the two probes have a small separation compared to the scale length of the potential variations, it can be argued that the signal approximates a component of the electric field in the direction of the line connecting the two probes. For large separations compared to the scale length, the signal can be interpreted as being filtered, as best illustrated by considering a plane wave. Let the electrostatic potential in space be given as

$\Psi(r, t)=A \sin \left(\boldsymbol{k}_{0} \cdot \boldsymbol{r}-\omega_{0} t+\phi_{0}\right)$,

where $\phi_{0}$ is a phase. The two probes are separated by $\boldsymbol{\Delta}$, and the difference signal divided by the probe separation becomes

$$
\begin{aligned}
\mathcal{S}_{E}(r, t) & =\frac{k_{\Delta}}{\boldsymbol{\Delta} \cdot \boldsymbol{k}_{0}}(\Psi(r+\boldsymbol{\Delta}, t)-\Psi(r, t)) \\
& =A k_{\Delta} \frac{\sin \left(\frac{1}{2} \boldsymbol{\Delta} \cdot \boldsymbol{k}_{0}\right)}{\frac{1}{2} \boldsymbol{\Delta} \cdot \boldsymbol{k}_{0}} \cos \left(\boldsymbol{k}_{0} \cdot \boldsymbol{r}-\omega_{0} t+\phi_{0}+\frac{\boldsymbol{\Delta} \cdot \boldsymbol{k}_{0}}{2}\right),
\end{aligned}
$$

where $k_{\Delta}$ is wave vector component along $\Delta$, so that we have $k_{\Delta} \equiv \boldsymbol{\Delta} \cdot \boldsymbol{k}_{0} /|\boldsymbol{\Delta}|$. For small $\boldsymbol{\Delta} \cdot \boldsymbol{k}_{0}$ we note that

$$
\frac{\sin \left(\frac{1}{2} \boldsymbol{\Delta} \cdot \boldsymbol{k}_{0}\right)}{\frac{1}{2} \boldsymbol{\Delta} \cdot \boldsymbol{k}_{0}} \approx 1
$$

and $\mathcal{S}_{E}(r, t)$ approximates the electric field derived from $\Psi(r, t)$ when obtained at at the centre-of-mass position, between the two detecting probes. For larger $\boldsymbol{\Delta} \cdot \boldsymbol{k}_{0}$ we can interpret

$$
\frac{\sin \left(\frac{1}{2} \boldsymbol{\Delta} \cdot \boldsymbol{k}_{0}\right)}{\frac{1}{2} \boldsymbol{\Delta} \cdot \boldsymbol{k}_{0}}
$$

as a "filter" that reduces the detected signal. If the rocket or satellite is spinning with a frequency $\Omega$ while detecting a plane wave with fixed phase velocity so that $\boldsymbol{\Delta} \cdot \boldsymbol{k}_{0}=$ $\Delta k_{0} \sin (\Omega t)$, the filter characteristic will be observable (Kelley and Mozer, 1973; Pfaff et al., 1997).

For a wide spectrum of waves, the situation is different. The conditions can best be illustrated by a model signal obtained by a random superposition of structures that represents the turbulence. Using a model in one spatial dimension for simplicity, we have

$\Psi(x, t)=\sum_{j} a_{j} \phi\left(x-x_{j}, t-t_{j}\right)$,

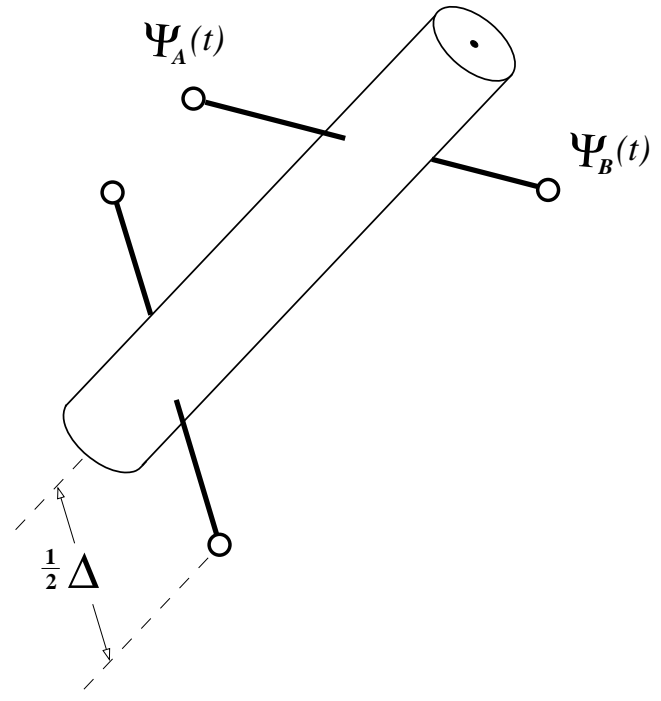

Figure A1. Schematic illustration of a standard example of probe positioning on a rocket (Rose et al., 1992). Altogether six probe potential difference signals can be obtained; two of them are redundant.

where $x_{j}, t_{j}$ is the random space-time position of a structure with randomly distributed amplitudes $a_{j}$. This model is standard and has been used many times. For a fully developed strong turbulence, we can let some structures represent small scales and other structures intermediate or large scales. By Campbell's theorem and its extensions (Campbell, 1909a, b; Rice, 1944; Pécseli, 2000), the autocorrelation function of the signal $\Psi(x, t)$ can be determined analytically as

$R_{\Psi}(\xi, \tau)=\mu\left\langle a^{2}\right\rangle \iint \phi(x, t) \phi(x-\xi, t-\tau) \mathrm{d} x \mathrm{~d} t$,

where $\mu$ is the density of structures $\phi(x, t)$ in the spacetime record and $\xi, \tau$ represent spatial and temporal separations in the idealized space-time-varying stationary and homogeneous signal record. We denote the correlation length of $R_{\Psi}(\xi, \tau)$ by $\ell_{\text {cor }}$ and the correlation time by $\tau_{\text {cor }}$.

For the probe difference signal (see Fig. A1)

$$
\begin{aligned}
\Psi_{A}-\Psi_{B}= & \sum_{j} a_{j}\left(\phi\left(x-x_{j}, t-t_{j}\right)\right. \\
& \left.-\phi\left(x+\Delta-x_{j}, t-t_{j}\right)\right),
\end{aligned}
$$

we find the autocorrelation function

$$
\begin{aligned}
R_{\mathcal{S}}(\xi, \tau)= & 2 R_{\Psi}(\xi, \tau) \\
& -R_{\Psi}(\xi+\Delta, \tau)-R_{\Psi}(\xi-\Delta, \tau) .
\end{aligned}
$$

We can divide $R_{\mathcal{S}}(\xi, \tau)$ by $\Delta^{2}$ and find for spatial separations $\Delta \ll \ell_{\text {cor }}$ the result $R_{\mathcal{S}}(\xi, \tau) / \Delta^{2} \approx-\partial^{2} R_{\Psi}(\xi, \tau) / \partial \xi^{2}$, i.e. the electric field correlation function, as expected. 
We now introduce a relative velocity $V_{\mathrm{R}}$ between the detector and the structures. The signal sampled along the trajectory $\xi=V_{\mathrm{R}} \tau$ will vary with time only. Basically, we find two cases as illustrated in Fig. A2. In one case $\Delta \ll \ell_{\text {cor }}$, and we detect a temporal signal corresponding to the electric field: here the scale sizes (or wavelengths) in the turbulence are much longer than the probe separation. The other limiting case with $\boldsymbol{\Delta} \gg \ell_{\text {cor }}$ has scale sizes (or wavelengths) in the turbulence shorter than the probe separation, and the two last terms in Eq. (A3) are clearly separated in space and time from the first one. Here the detector samples the correlation function for the potential, provided that the correlation time is smaller than $\Delta / V_{R}$. In this latter limiting case, one probe acts as a reference "ground" for the other one (Krane et al., 2000), and in this sense it is possible to detect potential fluctuations also by a rocket or satellite. For realistic conditions we have all scales represented at the same time, and the argument has to be rephrased: when the Taylor hypothesis is applicable, we generally expect that when they are present, low-frequency-long-wavelength components represent electric fields, while high frequencies and short wavelengths will approximate the electrostatic potential variations in the power spectra.

The projection of the separation vector $\Delta$ on the direction of propagation $\boldsymbol{V}_{\mathrm{R}}$ can vary with time due to a rocket or satellite spin. In such cases it might be necessary to sample data in segments corresponding to a selected spin phase in order to obtain spectra under uniform conditions (Krane et al., 2000).
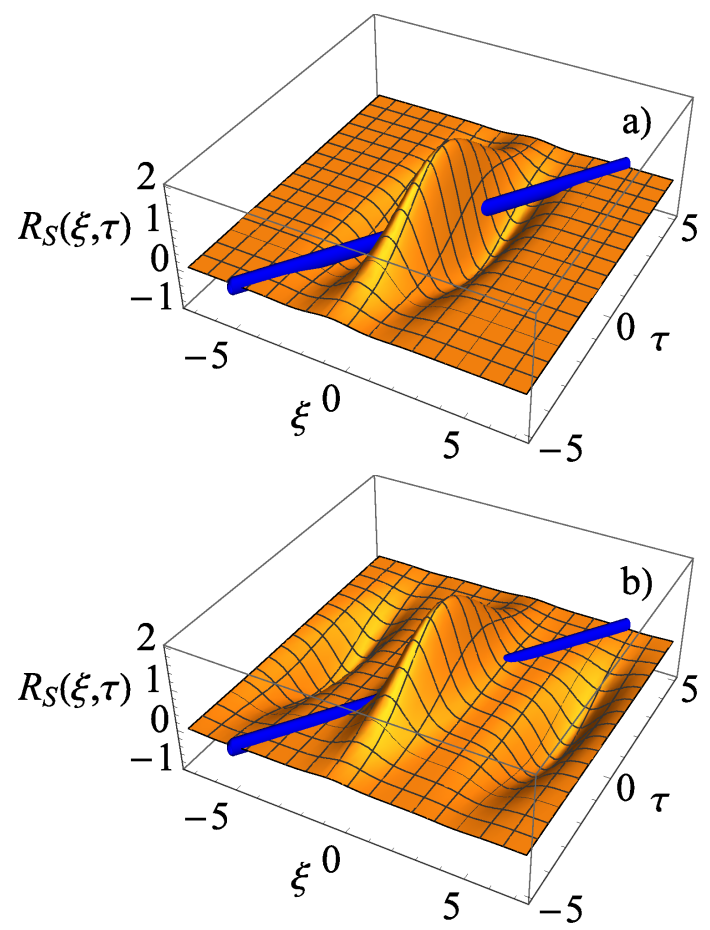

Figure A2. Two possible autocorrelation functions found by sampling a signal along a trajectory $\xi=V_{\mathrm{R}} \tau$, shown as a solid blue line. In the first case (a) with $\Delta \ll \ell_{\text {cor }}$ ) we detect the correlation function for the electric field; in the second case (b) with $\left.\Delta \gg \ell_{\text {cor }}\right)$, we find the correlation function for the electrostatic potential. The figure uses an illustrative model, with $R_{\Psi}(\xi, \tau)=$ $\exp \left(-\xi^{2} / \ell_{\text {cor }}^{2}-\tau^{2} / \tau_{\text {cor }}^{2}+\zeta \xi \tau /\left(\ell_{\text {cor }} \tau_{\text {cor }}\right)\right)$ with $\zeta=0.75$. 
Acknowledgements. The author acknowledges valuable discussions with Lars Dyrud, Bård Krane, Søren Larsen, Torben Mikkelsen, Chan-Mou Tchen, Jan Trulsen and Andrzej Wernik. Thanks also to Edward Powers for permission to reproduce Fig. 1. Bjørn Lybek offered valuable help with several figures.

The topical editor L. Blomberg thanks J. Borovsky and one anonymous referee for help in evaluating this paper.

\section{References}

Alport, M. J., D’Angelo, N., and Pécseli, H. L.: A laboratory experiment on EM backscatter from Farley-Buneman and gradient drift waves, J. Geophys. Res., 86, 7694-7702, doi:10.1029/JA086iA09p07694, 1981.

Angelopoulos, V., Mozer, F. S., Bonnell, J., Temerin, M., Somoza, M., Peterson, W. K., Collin, H. L., and Giles, B.: Wave power studies of cusp crossings with the Polar satellite, J. Geophys. Res.-Space, 106, 5987-6006, doi:10.1029/2000JA900127, 2001.

Basu, S., Basu, S., MacKenzie, E., Fougere, P. F., Coley, W. R., Maynard, N. C., Winningham, J. D., Sugiura, M., Hanson, W. B., and Hoegy, W. R.: Simultaneous density and electric field fluctuation spectra associated with velocity shears in the auroral oval, J. Geophys. Res., 93, 115-136, doi:10.1029/JA093iA01p00115, 1988.

Basu, S., Basu, S., MacKenzie, E., Coley, W. R., Sharber, J. R., and Hoegy, W. R.: Plasma structuring by the gradient drift instability at high latitudes and comparison with velocity shear driven processes, J. Geophys. Res., 95, 7799-7818, doi:10.1029/JA095iA06p07799, 1990.

Batchelor, G. K.: The Theory of Homogeneous Turbulence, Cambridge University Press, Cambridge, UK, 1953.

Beall, J. M., Kim, Y. C., and Powers, E. J.: Estimation of wavenumber and frequency spectra using fixed probe pairs, J. Applied Phys., 53, 3933-3940, doi:10.1063/1.331279, 1982.

Bekefi, G.: Radiation Processes in Plasmas, John Wiley and Sons, New York, USA, 1966.

Birkenmeier, G., Ramisch, M., Fuchert, G., Köhn, A., Nold, B., and Stroth, U.: Spatial structure of drift-wave turbulence and transport in a stellarator, Plasma Phys. Controlled Fusion, 55, 015003 , doi:10.1088/0741-3335/55/1/015003, 2013.

Bol, K.: Density Fluctuations in the Etude Stellarator, Phys. Fluids, 7, 1855-1863, doi:10.1063/1.2746786, 1964.

Bruno, R. and Carbone, V.: The solar wind as a turbulence laboratory, Living Rev. Solar Phys., 1-162, 2005.

Buckingham, E.: On physically similar systems; illustrations of the use of dimensional equations, Phys. Rev., 4, 345-376, doi:10.1103/PhysRev.4.345, 1914.

Buneman, O.: Excitation of field aligned sound waves by electron streams, Phys. Rev. Lett., 10, 285-287, doi:10.1103/PhysRevLett.10.285, 1963.

Campbell, N.: The study of discontinuous phenomena, Proc. Phil. Soc., 15, 117-136, 1909a.

Campbell, N.: Discontinuities in light emission, Proc. Phil. Soc., 15, 310-328, 1909b.

Cerisier, J. C., Berthelier, J. J., and Beghin, C.: Unstable density gradients in the high-latitude ionosphere, Radio Science, 20, 755761, doi:10.1029/RS020i004p00755, 1985.
Chandrasekhar, S.: The theory of statistical and isotropic turbulence, Phys. Rev., 75, 896-897, doi:10.1103/PhysRev.75.896, 1949.

Chandrasekhar, S.: The theory of turbulence, J. Madras Univ., B 27, 251-275, 1957.

Chekhlov, A., Orszag, S. A., Sukoriansky, S., Galperin, B., and Staroselsky, I.: The effect of small-scale forcing on largescale structures in two-dimensional flows, Physica D: Nonlinear Phenomena, 98, 321-334, doi:10.1016/0167-2789(96)00102-9, 1996.

Chen, F. F.: Spectrum of low- $\beta$ plasma turbulence, Phys. Rev. Lett., 15, 381-383, doi:10.1103/PhysRevLett.15.381, 1965a.

Chen, F. F.: Excitation of drift instabilities in thermionic plasmas, Plasma Phys. (J. Nucl. Energy Part C), 7, 399-417, doi:10.1088/0368-3281/7/4/305, 1965b.

Chen, F. F.: Effect of sheaths on drift instabilities in thermionic plasmas, Phys. Fluids, 8, 752-754, doi:10.1063/1.1761294, 1965c.

Chen, F. F.: Resistive overstabilities and anomalous "diffusion", Phys. Fluids, 8, 912-919, doi:10.1063/1.1761335, 1965d.

Chen, F. F.: "Universal" overstability of a resistive, inhomogeneous plasma, Phys. Fluids, 8, 1323-1333, doi:10.1063/1.1761403, 1965 e.

Chen, F. F.: Axial eigenmodes for long- $\lambda \|$ waves in plasmas bounded by sheaths, Phys. Fluids, 22, 2346-2358, doi:10.1063/1.862546, 1979.

Chen, F. F.: Introduction to Plasma Physics and Controlled Fusion, 2nd Edn., vol. 1, Plenum Press, New York, USA, 1984.

Constantin, P.: New Perspectives in Turbulence, Chap. 8: Remarks on the Navier-Stokes equations, Springer Verlag, Berlin, Germany, 1991.

Cowley, S. C., Kulsrud, R. M., and Sudan, R.: Considerations of ion-temperature-gradient-driven turbulence, Phys. Fluids B: Plasma Phys., 3, 2767-2782, doi:10.1063/1.859913, 1991.

D'Angelo, N.: On high-latitude CNA, F-lacuna and SEC, Ann. Geophys., 34, 51-54, 1978,

http://www.ann-geophys.net/34/51/1978/.

D'Angelo, N. and Enriques, L.: Turbulence of a low- $\beta$ plasma, Phys. Fluids, 9, 2290-2291, doi:10.1063/1.1761607, 1966.

D'Angelo, N. and von Goeler, S.: Investigation of the KelvinHelmholtz instability in a Cesium plasma, Phys. Fluids, 9, 309313, doi:10.1063/1.1761674, 1966.

D’Angelo, N., Pécseli, H. L., and Petersen, P. I.: Turbulence in a cusp Q device, Phys. Fluids, 17, doi:10.1063/1.1694629, 1974.

Diamond, P. H., Itoh, S.-I., and Itoh, K.: Modern Plasma Physics, vol. I: Physical Kinetics of Turbulent Plasmas, Cambridge University Press, Cambridge, UK, 2010.

Drazin, P. G. and Johnson, R. S.: Solitons: an Introduction, Cambridge University Press, Cambridge, UK, 1989.

Dupree, T. H.: Turbulence in Fluids and Plasmas, Chap. 1: Introduction to basic phenomena of turbulence in plasmas, Polytechnic Press, Brooklyn, NY, USA, 1969.

Dyrud, L., Krane, B., Oppenheim, M., Pécseli, H. L., Schlegel, K., Trulsen, J., and Wernik, A. W.: Low-frequency electrostatic waves in the ionospheric E-region: a comparison of rocket observations and numerical simulations, Ann. Geophys., 24, 29592979, doi:10.5194/angeo-24-2959-2006, 2006.

Dyson, P. L., McClure, J. P., and Hanson, W. B.: In situ measurements of the spectral characteristics of $\mathrm{F}$ region 
ionospheric irregularities, J. Geophys. Res., 79, 1497-1502, doi:10.1029/JA079i010p01497, 1974.

Dysthe, K. B., Misra, K. D., and Trulsen, J. K.: On the linear cross-field instability problem, J. Plasma Phys., 13, 249-257, doi:10.1017/S0022377800026027, 1975.

Earle, G. D., Kelley, M. C., and Ganguli, G.: Large velocity shears and associated electrostatic waves and turbulence in the auroral F region, J. Geophys. Res.-Space, 94, 15321-15333, doi:10.1029/JA094iA11p15321, 1989.

Farley, D. T.: Two-stream plasma instability as a source of irregularities in the ionosphere, Phys. Rev. Lett., 10, 279-282, doi:10.1103/PhysRevLett.10.279, 1963.

Fejer, B., Farley, D., Balsley, B., and Woodman, R.: Vertical Structure of the VHF backscattering region in the equatorial electrojet and the gradient drift instability, J. Geophys. Res., 80, 13131324, doi:10.1029/JA080i010p01313, 1975.

Foster, J. C., Tetenbaum, D., del Pozo, C. F., St-Maurice, J. P., and Moorcroft, D. R.: Aspect angle variations in intensity, phase velocity, and altitude for high-latitude $34-\mathrm{cm} \mathrm{E} \mathrm{re-}$ gion irregularities, J. Geophys. Res.-Space, 97, 8601-8617, doi:10.1029/91JA03144, 1992.

Fowler, T. K.: Plasma fluctuations and the control of pumpout, Phys. Fluids, 9, 1437-1441, doi:10.1063/1.1761876, 1966.

Fredricks, R. W. and Coroniti, F. V.: Ambiguities in the deduction of rest frame fluctuation spectrums from spectrums computed in moving frames, J. Geophys. Res., 81, 5591-5595, doi:10.1029/JA081i031p05591, 1976.

Frisch, U.: Turbulence: The Legacy of A. N. Kolmogorov, Cambridge University Press, Cambridge, UK, 1995.

Garcia, O. E. and Pécseli, H. L.: Models for electrostatic drift waves with density variations along magnetic field lines, Geophys. Res. Lett., 40, 5565-5569, doi:10.1002/2013GL057802, 2013.

Garcia, O. E., Leer, E., Pécseli, H. L., and Trulsen, J. K.: Magnetic field-aligned plasma currents in gravitational fields, Ann. Geophys., 33, 257-266, doi:10.5194/angeo-33-257-2015, 2015.

Golovchanskaya, I. V., Kozelov, B. V., and Despirak, I. V.: Investigation of the broadband ELF turbulence by observations of the FAST satellite, Geomagn. Aeronomy, 52, 474-481, doi:10.1134/S0016793212040044, 2012.

Gondarenko, N. A. and Guzdar, P. N.: Plasma patch structuring by the nonlinear evolution of the gradient drift instability in the high-latitude ionosphere, J. Geophys. Res.-Space, 109, A09301, doi:10.1029/2004JA010504, 2004.

Grant, H. L., Stewart, R. W., and Moilliet, A.: Turbulence spectra from a tidal channel, J. Fluid Mech., 12, 241-268, doi:10.1017/S002211206200018X, 1962.

Guio, P. and Pécseli, H. L.: Phase space structures generated by absorbing obstacles in streaming plasmas, Ann. Geophys., 23, 853-865, doi:10.5194/angeo-23-853-2005, 2005.

Gürcan, Ö. D., Garbet, X., Hennequin, P., Diamond, P. H., Casati, A., and Falchetto, G. L.: Wave-number spectrum of drift-wave turbulence, Phys. Rev. Lett., 102, 255002, doi:10.1103/PhysRevLett.102.255002, 2009.

Gurevich, A., Rinnert, K., and Schlegel, K.: Long wave part of the plasma turbulence spectrum in the lower e-region, Radiophys. Quantum Electronics, 40, 16-30, doi:10.1007/BF02677821, 1997.
Gurnett, D. A. and Frank, L. A.: A region of intense plasma wave turbulence on auroral field lines, J. Geophys. Res., 82, 10311050, doi:10.1029/JA082i007p01031, 1977.

Hamza, A. M. and St-Maurice, J. P.: A self-consistent fully turbulent theory of auroral E region irregularities, J. Geophys. Res.Space, 98, 11601-11613, doi:10.1029/92JA02835, 1993a.

Hamza, A. M. and St-Maurice, J.-P.: A turbulent theoretical framework for the study of current-driven $\mathrm{E}$ region irregularities at high latitudes: Basic derivation and application to gradient-free situations, J. Geophys. Res.-Space, 98, 1158711599, doi:10.1029/92JA02836, 1993 b.

Hamza, A. M. and St-Maurice, J. P.: A fully self-consistent fluid theory of anomalous transport in Farley-Buneman turbulence, J. Geophys. Res., 100, 9653-9668, doi:10.1029/94JA03031, 1995.

Hasegawa, A. and Mima, K.: Pseudo-three-dimensional turbulence in magnetized nonuniform plasma, Phys. Fluids, 21, 87-92, doi:10.1063/1.862083, 1978.

Hasegawa, A. and Wakatani, M.: Plasma edge turbulence, Phys. Rev. Lett., 50, 682-686, doi:10.1103/PhysRevLett.50.682, 1983.

Hatakeyama, R., Oertl, M., Märk, E., and Schrittwieser, R.: Collisionless drift instability and ion heating in a currentcarrying inhomogeneous plasma, Phys. Fluids, 23, 1774-1781, doi:10.1063/1.863203, 1980.

Hatakeyama, R., Moon, C., Tamura, S., and Kaneko, T.: Collisionless drift waves ranging from current-driven, shear-modified, and electron-temperature-gradient modes, Contrib. Plasma Phys., 51, 537-545, doi:10.1002/ctpp.201010156, 2011.

Heisenberg, W.: Zur statistischen Theorie der Turbulenz, Z. Phys., 124, 628-657, an english translation of Heisenberg's paper is available for instance as Nato Advisory Committee for Aeronautics, Technical Memorandum 1431, 1948.

Hendel, H. W., Chu, T. K., and Politzer, P. A.: Collisional drift waves - identification, stabilization, and enhanced plasma transport, Phys. Fluids, 11, 2426-2439, doi:10.1063/1.1691833, 1968.

Hennequin, P., Sabot, R., Honoré, C., Hoang, G. T., Garbet, X., Truc, A., Fenzi, C., and Quéméneur, A.: Scaling laws of density fluctuations at high-k on Tore Supra, Plasma Phys. Controlled Fusion, 46, B121, doi:10.1088/0741-3335/46/12B/011, 2004.

Hinze, J. O.: Turbulence, 2 Edn., McGraw Hill, New York, USA, 1975.

Horton, W.: Nonlinear drift waves and transport in magnetized plasma, Phys. Reports, 192, 1-177, doi:10.1016/03701573(90)90148-U, 1990.

Horton, W.: Drift waves and transport, Rev. Modern Phys., 71, 735778, doi:10.1103/RevModPhys.61.763, 1999.

Huld, T., Iizuka, S., Pécseli, H. L., and Rasmussen, J. J.: Experimental investigation of flute-type electrostatic turbulence, Plasma Phys. Control. Fusion, 30, 1297-1318, doi:10.1088/07413335/30/10/008, 1988.

Hysell, D.: An overview and synthesis of plasma irregularities in equatorial spread F, J. Atmos. Solar-Terrestrial Phys., 62, 10371056, doi:10.1016/S1364-6826(00)00095-X, 2000.

Iranpour, K., Pécseli, H. L., Trulsen, J., Bahnsen, A., Primdahl, F., and Rinnert, K.: Propagation and dispersion of electrostatic waves in the ionospheric E region, Ann. Geophys., 15, 878, 1997.

Iwama, N., Ohba, Y., and Tsukishima, T.: Estimation of wavenumber spectrum parameters from fixed probe-pair data, J. Applied Phys., 50, 3197-3206, doi:10.1063/1.326356, 1979. 
Jassby, D. L.: Transverse velocity shear instabilities within a magnetically confined plasma, Phys. Fluids, 15, 1590-1604, doi:10.1063/1.1694135, 1972.

Jiquan, L. and Kishimoto, Y.: Wave-number spectral characteristics of drift wave micro-turbulence with large-scale structures, Plasma Sci. Technology, 13, 297-301, doi:10.1088/10090630/13/3/06, 2011.

John, P. I. and Saxena, Y. C.: Observation of Farley-Buneman instability in laboratory plasma, Geophys. Res. Lett., 2, 251-254, doi:10.1029/GL002i006p00251, 1975.

Kadomtsev, B. B.: Plasma Turbulence, Academic Press, New York, USA, 1965.

Kelley, M. C.: Nonlinear saturation spectra of electric fields and density fluctuations in drift wave turbulence, Phys. Fluids, 25, 1002-1004, doi:10.1063/1.863854, 1982.

Kelley, M. C.: The Earth's Ionosphere, Plasma Physics and Electrodynamics, vol. 43 of International Geophysics Series, Academic Press, San Diego, California, USA, 1989.

Kelley, M. C. and Mozer, F. S.: Electric field and plasma density oscillations due to the high-frequency Hall current two-stream instability in the Auroral E region, J. Geophys. Res., 78, 2214 2221, doi:10.1029/JA078i013p02214, 1973.

Kelley, M. C., Livingston, R. C., Rino, C. L., and Tsunoda, R. T.: The vertical wave number spectrum of topside equatorial spread F: Estimates of backscatter levels and implications for a unified theory, J. Geophys. Res.-Space, 87, 5217-5221, doi:10.1029/JA087iA07p05217, 1982a.

Kelley, M. C., Pfaff, R., Baker, K. D., Ulwick, J. C., Livingston, R., Rino, C., and Tsunoda, R.: Simultaneous rocket probe and radar measurements of equatorial spread FTransitional and short wavelength results, J. Geophys. Res.-Space, 87, 1575-1588, doi:10.1029/JA087iA03p01575, 1982b.

Kent, G. I., Jen, N. C., and Chen, F. F.: Transverse KelvinHelmholtz instability in a rotating plasma, Phys. Fluids, 12, 2140-2151, doi:10.1063/1.1692323, 1969.

Keskinen, M. J. and Huba, J. D.: Nonlinear evolution of highlatitude ionospheric interchange instabilities with scale-sizedependent magnetospheric coupling, J. Geophys. Res.-Space, 95, 15157-15166, doi:10.1029/JA095iA09p15157, 1990.

Keskinen, M. J., Mitchell, H. G., Fedder, J. A., Satyanarayana, P., Zalesak, S. T., and Huba, J. D.: Nonlinear evolution of the Kelvin-Helmholtz instability in the highlatitude ionosphere, J. Geophys. Res.-Space,, 93, 137-152, doi:10.1029/JA093iA01p00137, 1988.

Kim, Y. C. and Powers, E. J.: Digital bispectral analysis and its applications to nonlinear wave interactions, IEEE Trans. Plasma Sci., PS-7, 120-131, doi:10.1109/TPS.1979.4317207, 1979.

Kintner, P. M.: Observations of velocity shear driven plasma turbulence, J. Geophys. Res., 81, 5114-5122, doi:10.1029/JA081i028p05114, 1976.

Kintner, P. M. and Seyler, C. E.: The status of observations and theory of high latitude ionospheric and magnetospheric plasma turbulence, Space Sci. Rev., 41, 91-129, doi:10.1007/BF00241347, 1985.

Koepke, M. E.: Interrelated laboratory and space plasma experiments, Rev. Geophys., 46, RG3001, doi:10.1029/2005RG000168, 2008.

Koepke, M. E. and Reynolds, E. W.: Simultaneous, co-located parallel-flow shear and perpendicular-flow shear in a low- temperature, ionospheric-plasma relevant laboratory plasma, Plasma Phys. Contr. Fusion, 49, A145-A157, doi:10.1088/07413335/49/5A/S12, 2007.

Kraichnan, R. H.: Inertial ranges in two-dimensional turbulence, Phys. Fluids, 10, 1417-1423, doi:10.1063/1.1762301, 1967.

Kraichnan, R. H.: Inertial-range transfer in two- and threedimensional turbulence, J. Fluid Mech., 47, 525-535, doi:10.1017/S0022112071001216, 1971.

Krane, B., Pécseli, H. L., Trulsen, J., and Primdahl, F.: Spectral properties of low-frequency electrostatic waves in the ionospheric E region, J. Geophys. Res., 105, 10585-10601, doi:10.1029/1999JA900503, 2000.

LaBelle, J., Kelley, M. C., and Seyler, C. E.: An analysis of the role of drift waves in equatorial spread F, J. Geophys. Res.-Space, 91, 5513-5525, doi:10.1029/JA091iA05p05513, 1986.

Larsen, Y., Hanssen, A., Krane, B., Pécseli, H. L., and Trulsen, J.: Time-resolved statistical analysis of nonlinear electrostatic fluctuations in the ionospheric E region, J. Geophys. Res., 107, SIA5-1 - SIA5-11, doi:10.1029/2001JA900125, 2002.

Latten, A., Klinger, T., Piel, A., and Pierre, T.: A probe array for the investigation of spatio-temporal structures in drift wave turbulence, Rev. Scientific Instr., 66, 3254-3262, doi:10.1063/1.1145490, 1995.

Levinson, S. J., Beall, J. M., Powers, E. J., and Bengtson, R. D.: Space/time statistics of the turbulence in a tokamak edge plasma, Nuclear Fusion, 24, 527-539, doi:10.1088/0029-5515/24/5/001, 1984.

Liewer, P. C.: Measurements on microturbulence in Tokamaks and comparison with theories of turbulence and anomalous transport, Nucl. Fusion, 25, 543-621, doi:10.1088/0029-5515/25/5/004, 1985.

Lilly, D. K.: Numerical simulation of two-dimensional turbulence, Phys. Fluids, 12, II-240-II-249, doi:10.1063/1.1692444, 1969.

Lynov, J. P., Nielsen, A. H., Pécseli, H. L., and Juul Rasmussen, J.: Studies of the Eulerian-Lagrangian transformation in twodimensional random flows, J. Fluid Mech., 224, 485-505, doi:10.1017/S0022112091001842, 1991.

Machalek, M. D. and Nielsen, P.: Light-scattering measurements of turbulence in a normal shock, Phys. Rev. Lett., 31, 439-442, doi:10.1103/PhysRevLett.31.439, 1973.

Maggs, J. E. and Morales, G. J.: Exponential power spectra, deterministic chaos and Lorentzian pulses in plasma edge dynamics, Plasma Phys. Controlled Fusion, 54, 124041, doi:10.1088/07413335/54/12/124041, 2012.

McDaniel, R. D. and Hysell, D. L.: Models and DE II observations of inertial-regime irregularities in equatorial spread F, J. Geophys. Res.-Space, 102, 22233-22246, doi:10.1029/97JA02038, 1997.

McDonald, B. E., Coffey, T. P., Ossakow, S., and Sudan, R. N.: Preliminary report of numerical simulation of type 2 irregularities in the equatorial electrojet, J. Geophys. Res., 79, 2551-2554, doi:10.1029/JA079i016p02551, 1974.

Mikkelsen, T. and Pécseli, H. L.: Investigations of strong turbulence in a low- $\beta$ plasma, Phys. Rev. Lett., 41, 951, doi:10.1103/PhysRevLett.41.951, 1978.

Mikkelsen, T. and Pécseli, H. L.: Strong turbulence in partially ionized plasmas, Phys. Lett. A, 77, 159-162, doi:10.1016/03759601(80)90179-6, 1980. 
Mikkelsen, T., Larsen, S. E., and Pécseli, H. L.: Diffusion of Gaussian puffs, Quart. J. Roy. Meteorol. Soc., 113, 81-105, doi:10.1256/smsqj.47505, 1987.

Misguich, J. H., Balescu, R., Pécseli, H. L., Mikkelsen, T., Larsen, S. E., and Xiaoming, Q.: Diffusion of charged particles in turbulent magnetoplasmas, Plasma Phys. Contr. Fusion, 29, 825-856, doi:10.1088/0741-3335/29/7/004, 1987.

Motley, R. W.: Q Machines, Academic Press, New York, USA, 1975.

Mozer, F. S.: Power spectra of the magnetospheric electric field, J. Geophys. Res., 76, 3651-3667, doi:10.1029/JA076i016p03651, 1971.

Nagashima, Y., Itoh, S.-I., Shinohara, S., Fukao, M., Fujisawa, A., Terasaka, K., Kawai, Y., Kasuya, N., Tynan, G. R., Diamond, P. H., Yagi, M., Inagaki, S., Yamada, T., and Itoh, $\mathrm{K}$.: Coexistence of zonal flows and drift-waves in a cylindrical magnetized plasma, J. Physical Soc. Japan, 77, 114501, doi:10.1143/JPSJ.77.114501, 2008.

Nielsen, A. H., Pécseli, H. L., and Juul Rasmussen, J.: Vortex structures generated by the Kelvin-Helmholtz instability, Ann. Geophys., 10, 655-667, 1992,

http://www.ann-geophys.net/10/655/1992/

Odajima, K.: Effects of radial electric-field on drift wave instability in a weakly ionized plasma, J. Phys. Soc. Jap., 44, 1685-1693, doi:10.1143/JPSJ.44.1685, 1978.

Okabayashi, M. and Arunasalam, V.: Study of drift-wave turbulence by microwave scattering in a toroidal plasma, Nucl. Fusion, 17, 497-513, doi:10.1088/0029-5515/17/3/010, 1977.

Ott, E. and Farley, D. T.: The $k$ spectrum of ionospheric irregularities, J. Geophys. Res., 79, 2469-2472, doi:10.1029/JA079i016p02469, 1974.

Pécseli, H. L.: Drift-wave turbulence in low- $\beta$ plasmas, Phys. Scripta, T2/1, 147-157, doi:10.1088/0031-8949/1982/T2A/018, 1982.

Pécseli, H. L.: Fluctuations in Physical Systems, Cambridge University Press, Cambridge, UK, 2000.

Pécseli, H. L.: Waves and Oscillations in Plasmas, Taylor \& Francis, London, UK, 2012.

Pécseli, H. L. and Trulsen, J.: On the interpretation of experimental methods for investigating nonlinear wave phenomena, Plasma Phys. Contr. Fusion, 35, 1701-1715, doi:10.1088/07413335/35/12/004, 1993.

Pécseli, H. L., Mikkelsen, T., and Larsen, S. E.: Drift wave turbulence in a low- $\beta$ plasma, Plasma Phys., 25, 1173-1197, doi:10.1088/0032-1028/25/11/001, 1983.

Pécseli, H. L., Primdahl, F., and Bahnsen, A.: Low-frequency electrostatic turbulence in the polar cap E region, J. Geophys. Res., 94, 5337-5349, doi:10.1029/JA094iA05p05337, 1989.

Pécseli, H. L., Trulsen, J., Primdahl, F., and Bahnsen, A.: Propagation and nonlinear interaction of low-frequency electrostatic waves in the polar cap E region, J. Geophys. Res., 98, 16031612, doi:10.1029/92JA01403, 1993.

Pfaff, R. F., Kelley, M. C., Kudeki, E., Fejer, B. G., and Baker, K. D.: Electric field and plasma density measurements in the strongly driven daytime equatorial electrojet: 1 . The unstable layer and gradient drift waves, J. Geophys. Res.-Space, 92, 13578-13596, doi:10.1029/JA092iA12p13578, 1987a.

Pfaff, R. F., Kelley, M. C., Kudeki, E., Fejer, B. G., and Baker, K. D.: Electric field and plasma density measure- ments in the strongly driven daytime equatorial electrojet: 2. Two-stream waves, J. Geophys. Res.-Space, 92, 13597-13612, doi:10.1029/JA092iA12p13597, 1987b.

Pfaff, R. F., Marionni, P. A., and Swartz, W. E.: Wavevector observations of the two-stream instability in the daytime equatorial electrojet, Geophys. Res. Lett., 24, 1663-1666, doi:10.1029/97GL01535, 1997.

Prakash, S., Subbaraya, B. H., and Gupta, S. P.: A study of the equatorial E-region during evening twilight using a Langmuir probe, J. Atmos. Terrestrial Phys., 30, 1193-1202, doi:10.1016/S00219169(68)80007-8, 1968.

Prakash, S., Gupta, S. P., and Subbaraya, B. H.: Irregularities in the equatorial E region over Thumba, Radio Sci., 4, 791-796, doi:10.1029/RS004i009p00791, 1969.

Prakash, S., Gupta, S. P., Subbaray, B. H., and Jain, C. L.: Electrostatic plasma instabilities in the equatorial electrojet, NaturePhysical Sciences, 233, 56-58, doi:10.1038/physci233056a0, 1971.

Prasad, G., Bora, D., and Saxena, Y. C.: Spectral characteristics of low-frequency turbulence in toroidal collisional magnetoplasma, Phys. Plasmas, 1, 2519-2524, doi:10.1063/1.870579, 1994.

Rice, S. O.: Mathematical analysis of random noise, I, Bell System Tech. J., 23, 282-332, 1944.

Rinnert, K.: Plasma waves observed in the Auroral E region ROSE campaign, J. Atmos. Terr. Phys., 54, 683-692, doi:10.1016/00219169(92)90106-U, 1992.

Ritz, C. P., Brower, D. L., Rhodes, T. L., Bengtson, R. D., Levinson, S. J., Jr., N. C. L., Peebles, W. A., and Powers, E. J.: Characterization of tokamak edge turbulence by far-infrared laser scattering and Langmuir probes, Nuclear Fusion, 27, 1125-1134, doi:10.1088/0029-5515/27/7/006, 1987.

Robinson, D. C. and Rusbridge, M. G.: Structure of turbulence in the Zeta plasma, Phys. Fluids, 14, 2499-2511, doi:10.1063/1.1693359, 1971.

Rodrigues, F. S., Kelley, M. C., Roddy, P. A., Hunton, D. E., Pfaff, R. F., de La Beaujardiére, O., and Bust, G. S.: C/NOFS observations of intermediate and transitional scale-size equatorial spread F irregularities, Geophys. Res. Lett., 36, L00C05, doi:10.1029/2009GL038905, 2009.

Rogers, K. C. and Chen, F. F.: Direct measurements of drift wave growth rates, Phys. Fluids, 13, 513-516, doi:10.1063/1.1692947, 1970.

Rollefson, J. P.: On Kolmogorov's theory of turbulence and intermittency, Can. J. Phys., 56, 1426-1441, 1978.

Rose, G., Schlegel, K., Rinnert, K., Kohl, H., Nielsen, E., Dehmel, G., Friker, A., Lubken, F. J., Lühr, H., Neske, E., and Steinweg, A.: The ROSE project - scientific objectives and discussion of $1 \mathrm{st}$ results, J. Atmos. Terr. Phys., 54, 657-667, doi:10.1016/00219169(92)90104-S, 1992.

Roth, J. R.: Experimental study of spectral index, mode coupling, and energy cascading in a turbulent, hot-ion plasma, Phys. Fluids, 14, 2193-2202, doi:10.1063/1.1693311, 1971.

Roth, J. R., Krawczonek, W. M., Powers, E. J., Kim, Y. C., and Hong, J. Y.: Fluctuations and turbulence in an electric field bumpy torus plasma, J. Applied Phys., 52, 2705-2713, doi:10.1063/1.329078, 1981.

Rowberg, R. E. and Wong, A. Y.: Collisional drift waves in the linear regime, Phys. Fluids, 13, 661-671, doi:10.1063/1.1692973, 1970. 
Røyrvik, O. and Smith, L. G.: Comparison of mesospheric VHF radar echoes and rocket probe electron concentration measurements, J. Geophys. Res.-Space, 89, 9014-9022, doi:10.1029/JA089iA10p09014, 1984.

Rutgers, M. A.: Forced 2D turbulence: experimental evidence of simultaneous inverse energy and forward enstrophy cascades, Phys. Rev. Lett., 81, 2244-2247, doi:10.1103/PhysRevLett.81.2244, 1998.

Saxena, Y. C. and John, P. I.: Observation of the cross-field instability in laboratory plasma, Geophys. Res. Lett., 2, 492-495, doi:10.1029/GL002i011p00492, 1975.

Schlitt, L. G. and Hendel, H. W.: Effects of parallel wavelength on the collisional drift instability, Phys. Fluids, 15, 1578, doi:10.1063/1.1694134, 1972.

Shkarofsky, I. P.: Turbulence in Fluids and Plasmas, Chap. 21: Analytic forms for decaying turbulence functions, Polytechnic Press, Brooklyn, NY, USA, 1969.

Shume, E. B. and Hysell, D. L.: Spectral analysis of plasma drift measurements from the AE-E satellite: evidence of an inertial subrange in equatorial spread F, J. Atmospheric Solar-Terrestrial Phys., 66, 57-65, doi:10.1016/j.jastp.2003.08.005, 2004.

Smith, D. E. and Powers, E. J.: Experimental determination of the spectral index of a turbulent plasma from digitally computed power spectra, Phys. Fluids, 16, 1373-1374, doi:10.1063/1.1694524, 1973.

Sreenivasan, K. R.: On the universality of the Kolmogorov constant, Phys. Fluids, 7, 2778, doi:10.1063/1.868656, 1995.

Sulem, C., Sulem, P.-L., and Frisch, H.: Tracing complex singularities with spectral methods, J. Comp. Phys., 50, 138-161, doi:10.1016/0021-9991(83)90045-1, 1983.

Taylor, J. B. and McNamara, B.: Plasma diffusion in two dimensions, Phys. Fluids, 14, 1492-1499, doi:10.1063/1.1693635, 1971.

Tchen, C.-M.: On the spectrum of energy in turbulent shear flow, J. Res. Bur. Stand., 50, 51-62, 1953.

Tchen, C.-M.: Repeated-cascade theory of turbulence in an inhomogeneous plasma, Phys. Rev. A, 8, 500-514, doi:10.1103/PhysRevA.8.500, 1973.

Tchen, C.-M.: Cascade theory of turbulence in a stratified medium1, Tellus, 27, 1-14, doi:10.1111/j.2153-3490.1975.tb01649.x, 1975.

Tchen, C.-M. and Pierson, W. J.: A group-kinetic theory with a closure by memory loss for modeling turbulence in the atmosphere and the oceans, Meteorol. Atmos. Phys., 38, 95-105, doi:10.1007/BF01029951, 1988.
Tchen, C.-M., Pécseli, H. L., and Larsen, S. E.: Strong turbulence in low- $\beta$ plasmas, Plasma Phys., 23, 817-829, doi:10.1088/00321028/22/8/005, 1980.

Tennekes, H.: Eulerian and Lagrangian time microscales in isotropic turbulence, J. Fluid Mech., 67, 561-567, doi:10.1017/S0022112075000468, 1975.

Tennekes, H. and Lumley, J. L.: A First Course in Turbulence, The MIT press, Cambridge, Massachusetts, USA, 1972.

Truc, A., Quéméneur, A., Hennequin, P., Grésillon, D., Gervais, F., Laviron, C., Olivain, J., Saha, S. K., and Devynck, P.: ALTAIR: An infrared laser scattering diagnostic on the TORE SUPRA tokamak, Rev. Sci. Instruments, 63, 3716-3724, doi:10.1063/1.1143603, 1992.

Tu, C.-Y. and Marsch, E.: MHD structures, waves and turbulence in the solar wind: Observations and theories, Space Science Rev., 73, 1-210, doi:10.1007/BF00748891, 1995.

Tynan, G. R., Holland, C., Yu, J. H., James, A., Nishijima, D., Shimada, M., and Taheri, N.: Observation of turbulent-driven shear flow in a cylindrical laboratory plasma device, Plasma Phys. Controlled Fusion, 48, S51, doi:10.1088/0741-3335/48/4/S05, 2006.

Tynan, G. R., Fujisawa, A., and McKee, G.: A review of experimental drift turbulence studies, Plasma Phys. Controlled Fusion, 51, 113001, doi:10.1088/0741-3335/51/11/113001, 2009.

Weiland, J.: Collective Modes in Inhomogeneous Plasma, Kinetic and Advanced Fluid Theory, Institute of Physics Publishing, Bristol, UK, 2000.

Wootton, A. J., Carreras, B. A., Matsumoto, H., McGuire, K., Peebles, W. A., Ritz, C. P., Terry, P. W., and Zweben, S. J.: Fluctuations and anomalous transport in tokamaks, Phys. Fluids B, 2, doi:10.1063/1.859358, 1990.

Yamada, T., Itoh, S.-I., Maruta, T., Kasuya, N., Nagashima, Y., Shinohara, S., Terasaka, K., Yagi, M., Inagaki, S., Kawai, Y., Fujisawa, A., and Itoh, K.: Anatomy of plasma turbulence, Nature Phys., 4, 721-725, doi:10.1038/nphys1029, 2008.

Yamada, T., Itoh, S.-I., Inagaki, S., Nagashima, Y., Shinohara, S., Kasuya, N., Terasaka, K., Kamataki, K., Arakawa, H., Yagi, M., Fujisawa, A., and Itoh, K.: Two-dimensional bispectral analysis of drift wave turbulence in a cylindrical plasma, Phys. Plasmas, 17, 052313, doi:10.1063/1.3429674, 2010. 